\title{
Pilot Designs for Channel Estimation of MIMO OFDM Systems with Frequency-Dependent I/Q Imbalances
}

\author{
Hlaing Minn, Senior Member, IEEE, and Daniel Munoz, Student Member, IEEE
}

\begin{abstract}
Multiple input multiple output (MIMO) orthogonal frequency division multiplexing (OFDM) systems facilitate high data rate wireless communications, and require reliable channel estimates to fully materialize their advantages. The semiconductor downscaling trend has exacerbated device impairments such as inphase and quadrature (I/Q) imbalances which cause inter-carrier interferences in OFDM systems which cannot be remedied by increasing signal power. Different $R F$ chains of MIMO branches can cause different I/Q imbalances which further complicates MIMO OFDM channel estimation. This paper proposes several pilot designs for the estimation of the combined responses of MIMO frequency-selective channels and frequency-dependent $I / Q$ imbalances. The proposed designs require much smaller pilot overhead than the existing designs, and also provide estimation mean-squares error optimality (under white noise) and general applicability to preamble as well as pilot-data-multiplexed symbols in MIMO systems with or without null guard tones. Performance analyses and simulation results corroborate advantages of the proposed designs.
\end{abstract}

Index Terms-Channel estimation, I/Q imbalance, MIMO, OFDM, pilot design.

\section{INTRODUCTION}

$\mathbf{T}$ HE advances in semiconductor down-scaling have enabled a substantial growth of signal processing power but exacerbated the variations of the device characteristics due to more difficult control in the fabrication process [1]. The inphase (I) and quadrature (Q) imbalance, which represents mismatch between the I and $\mathrm{Q}$ branches, is a major impairment especially for direct-conversion transceivers with a high modulation order [2]. The higher data rate of nextgeneration wireless systems in a typical bandwidth limited regime requires $M$-ary quadrature amplitude modulation $(M$ QAM) with a large $M$. Both the semiconductor down-scaling trend and the need of a high modulation order underline the importance of I/Q imbalance issue for next generation wireless systems.

A viable transmission technology for current and next generation wireless systems is OFDM, while MIMO systems have

Paper approved by G. Bauch, the Editor for MIMO, Coding and Relaying of the IEEE Communications Society. Manuscript received March 12, 2009; revised July 23, 2009 and November 9, 2009.

The result for SISO systems was presented at IEEE WCNC 2009, the [TDM; Null] design at IEEE VTC 2009 (Fall), and [CDM-F; Null], [FDM; Null], [CDM-T; C-T] without Self-Mirror Tones, and [CDM-F or FDM; C-T] designs at IEEE Globecom 2009.

The authors are with the Dept. of Electrical Engineering, University of Texas at Dallas, Richardson, TX 75083 USA (e-mail: \{hlaing.minn, djm072000\}@utdallas.edu).

Digital Object Identifier 10.1109/TCOMM.2010.08.090150 become instrumental in providing higher data rate and better reliability in diverse wireless channels. The I/Q impairment in OFDM causes inter-carrier interferences. In MIMO OFDM, due to different RF chains for different antennas, I/Q imbalances may differ across antennas as well as across frequency (especially for ultra-wideband systems) for each antenna. For data detection, the multitudes of I/Q imbalance and channel parameters or their combined responses need to be estimated and compensated (see [3]-[9] for representative I/Q imbalance compensation methods). An efficient and practical way for the estimation is to transmit pilot tones. The main issue is how to design these pilots while considering the interferences among mirror tones and different transmit antennas as well as the estimation performance and overhead. The pilot designs may depend on whether they are developed for separate estimation of I/Q imbalances and channel responses or estimation of the combined responses. We consider the latter.

There are several pilot or training signal designs for MIMO OFDM channel estimation without I/Q imbalance (e.g., [10][14]). There also exist several pilot designs for I/Q imbalance estimation. The work in [15] applies two OFDM training symbols to perform per-subcarrier estimation of frequencydependent (FD) I/Q imbalance in single input single output (SISO) systems. The first training symbol has null tones at all negative subcarrier indexes and the second symbol contains null tones at all positive subcarrier indexes. This method neither optimizes the pilot overhead (large overhead) nor considers pilot-data-multiplexed symbols, MIMO OFDM, and exploitation of frequency-domain correlation. Similar drawbacks apply to [16] except it considers (mainly frequencyindependent (FI)) transmitter I/Q imbalance only, and uses a different pilot design. It uses an even number of OFDM training symbols with non-zero pilots where the pilots at the negative (positive) subcarrier indexes of the even symbols are the same as (negatives of) the corresponding pilots at the odd training symbols. The same pilot design using two OFDM training symbols is applied in [17] for the FI receiver I/Q imbalance, but [17] requires an additional training symbol for channel estimation, resulting in a larger overhead. It considers pilot-data-multiplexed symbols, but the other drawbacks still hold. [18] applies a pilot design (a combination of the designs in [15] and [16]) which uses an even number of OFDM training symbols with null pilots at the negative subcarrier indexes of the first half of OFDM training symbols and at the positive subcarrier indexes of the second half of OFDM 
training symbols. It considers FI receiver I/Q imbalance only, and is also associated with the above drawbacks.

For estimating the frequency-domain combined responses of the channel and FD I/Q imbalances, [19] presents two pilot designs using two OFDM training symbols. In the first design, the second OFDM training symbol is the 90 degree phase rotated version of the first symbol. The second design is the same as the one in [16]. It also provides a design condition for multiple OFDM symbols. But it uses a large overhead, addresses neither pilot-data-multiplexed symbols nor MIMO OFDM, and does not exploit frequency-domain correlation.

In [20], the authors consider a time-domain estimation which exploits frequency-domain correlation. It transmits equal-amplitude pilots on all subcarriers of one OFDM symbol. The mirror-tone interference is cancelled by designing the sum of the phases of the $q$ th pilot and its mirror tone to be $q \pi$. In this design the phases of the pilots at tone indexes 0 and $N / 2$ cannot be arbitrary as opposed to the statement in [20]. Moreover, when null guard tones are added at the band edges as in [21], the estimation optimality is no longer maintained. It does not address for pilot-data-multiplexed symbols and MIMO OFDM systems, and uses a large overhead. The pilot design in [22] uses all subcarriers of an OFDM symbol for pilots (large overhead), and is not applicable to systems with a pilot-data multiplexed format and/or with null guard tones. It only considers FI I/Q imbalance and requires a two step approach of estimation of I/Q parameters and compensation.

All of the above pilot designs for I/Q imbalance address for SISO OFDM systems only. Recently, [23] proposes a pilot design for the transmitter and receiver FI I/Q imbalances in MIMO-OFDM systems. Its extension to the FD I/Q imbalance is considered in [24]. Their pilot design uses the design in [16] with two OFDM training symbols as a basic block which is repeated according to Hadamard sequences for multiple transmit antennas. For $N_{\mathrm{Tx}}$ transmit antennas, they require $2 N_{\mathrm{Tx}}$ OFDM training symbols, and hence a large pilot overhead. Moreover, they are not applicable to pilot-data-multiplexed symbols, and do not provide the estimation optimality since the frequency-domain correlation is not exploited. A pilot design for space-time block coded systems in single-carrier frequency-flat fading channels is recently proposed in [25]. But it uses a large pilot overhead (at least two space-time code blocks), lacks the estimation optimality, and cannot be applied to systems with more than two transmit antennas.

In brief, the existing pilot designs for MIMO OFDM channel estimation do not consider the I/Q imbalance effects, while the existing pilot designs for the I/Q imbalance estimation do not address the channel estimation optimality. All of the latter designs require large pilot overheads, and most of them cannot be applied to MIMO systems and pilot-datamultiplexed symbols. In contrast, in this paper, we propose several pilot designs for MIMO OFDM equivalent channel estimation in the presence of transmitter and receiver FD I/Q imbalances, which avoid the drawbacks of the existing methods. Our designs require much less pilot overhead, and are more general and white-noise optimal in estimation.

The paper is organized as follows. Section II describes the signal model and Section III presents pilot design criteria. In Section IV, we study the relationships of the design criteria to

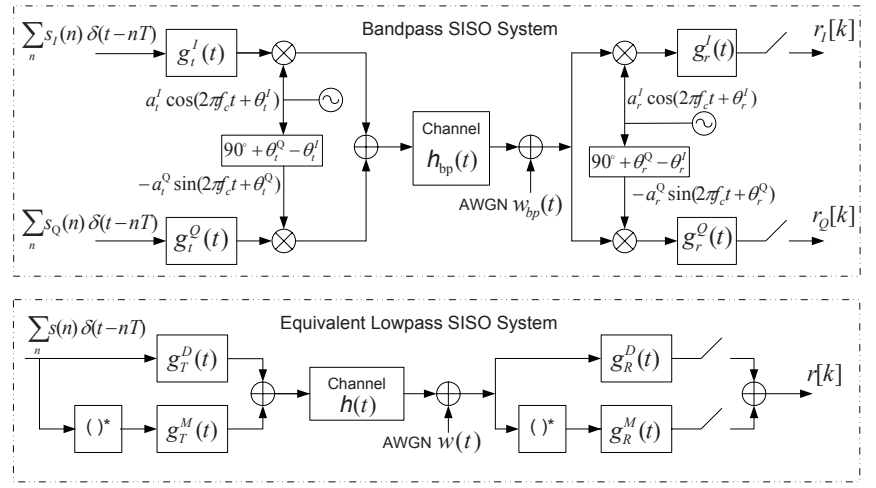

Fig. 1. Bandpass and equivalent lowpass SISO system models.

pilot symbols and structures, and propose several pilot designs. Performance evaluation and simulation results are discussed in Section V, and conclusions are provided in Section VI.

Notation: A bold capital (small) letter represents a matrix (a column vector). The superscripts $*, T$, and $H$ represent the conjugate, the transpose, and the conjugate transpose operations, respectively. $[\boldsymbol{X}]_{k, m}$ denotes the $k$-th row, $m$-th column element of $\boldsymbol{X}$. All indices start from 0 . The allone (all-zero) square matrix of size- $k$, the $k \times m$ all-zero matrix, and the $k \times k$ identity matrix are denoted by $\mathbf{1}_{k}\left(\mathbf{0}_{k}\right)$, $\mathbf{0}_{k \times m}$, and $\boldsymbol{I}_{k}$, respectively. $\operatorname{Tr}[\boldsymbol{X}]$ denotes the trace of $\boldsymbol{X}$. $\operatorname{diag}\{\boldsymbol{x}\}$ represents a diagonal matrix whose diagonal elements are defined by $\boldsymbol{x}$. The $l$-cyclic-forward-shifted version of $\boldsymbol{c}$ is denoted by $c^{(l)}$.* and $\otimes$ denote the convolution and the Kronecker product, respectively. $(l)_{N}$ represents $l$ modulo $N$. $\lfloor X\rfloor$ denotes the integer part of $X$ while $\lceil X\rceil$ represents the smallest integer greater than or equal to $X . \boldsymbol{F}$ denotes the $N$-point unitary discrete Fourier transform matrix and $\boldsymbol{f}_{k}$ is the $k$-th column of $\boldsymbol{F}$. The subcarrier indexes are modulo $N$ where $N$ is the total number of OFDM subcarriers. We will often use $-k$ to denote the subcarrier index $(-k)_{N}$, and $\&$ to represent the logical "and" operation.

\section{Signal MODEL}

First, consider a single antenna system with FI and FD I/Q imbalances at both transmitter and receiver sides as shown in Fig. 1 where $\left\{a_{t}^{I}, a_{t}^{Q}\right\}$ and $\left\{\theta_{t}^{I}, \theta_{t}^{Q}\right\}$ represent the FI gains and phase offsets of the I and Q branches at the transmitter (see [1]). The equivalent pulse shaping filters (i.e., the overall impulse responses including DAC, amplifier, pulse shaping, and FD I/Q imbalances) for the $\mathrm{I}$ and $\mathrm{Q}$ branches of the transmitter are denoted by $g_{t}^{I}(t)$ and $g_{t}^{Q}(t)$. These filters include effects of the FD I/Q imbalances [4], [21]. The receiver side parameters are defined similarly with the subscript $t$ replaced by $r$. An equivalent low-pass system is also presented in Fig. 1. The channel impulse response $h(t)$ is the lowpass-equivalent of the bandpass channel $h_{\mathrm{bp}}(t)$. The transmit system with I/Q imbalance can be viewed as the summation of two systems namely the direct system whose input is the same as the transmitter input signal $s(t)=s_{I}(t)+j s_{Q}(t)$ and the mirror system whose input is $s^{*}(t)$. The impulse responses of the direct and mirror systems at the transmitter are denoted by $g_{T}^{D}(t)$ and $g_{T}^{M}(t)$, and those at the receiver are represented 


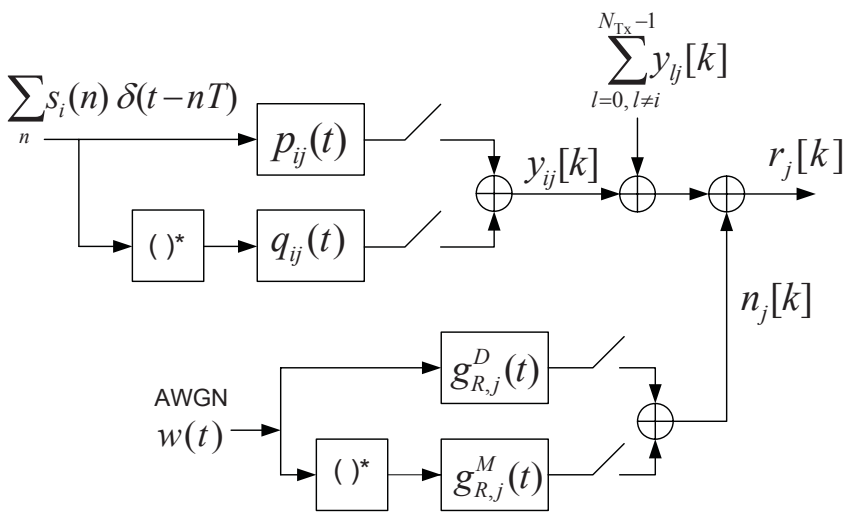

Fig. 2. A simplified equivalent lowpass MIMO system model.

by $g_{R}^{D}(t)$ and $g_{R}^{M}(t)$. Then, they can be given as

$$
\begin{aligned}
g_{T}^{D}(t) & =\frac{1}{2}\left[a_{t}^{I} e^{j \theta_{t}^{I}} g_{t}^{I}(t)+a_{t}^{Q} e^{j \theta_{t}^{Q}} g_{t}^{Q}(t)\right] \\
g_{T}^{M}(t) & =\frac{1}{2}\left[a_{t}^{I} e^{j \theta_{t}^{I}} g_{t}^{I}(t)-a_{t}^{Q} e^{j \theta_{t}^{Q}} g_{t}^{Q}(t)\right] \\
g_{R}^{D}(t) & =\frac{1}{2}\left[a_{r}^{I} e^{-j \theta_{r}^{I}} g_{r}^{I}(t)+a_{r}^{Q} e^{-j \theta_{r}^{Q}} g_{r}^{Q}(t)\right] \\
g_{R}^{M}(t) & =\frac{1}{2}\left[a_{r}^{I} e^{j \theta_{r}^{I}} g_{r}^{I}(t)-a_{r}^{Q} e^{j \theta_{r}^{Q}} g_{r}^{Q}(t)\right] .
\end{aligned}
$$

In MIMO systems, different RF chains for different antennas may give rise to different I/Q imbalances. The above responses corresponding to the $i$ th transmit antenna and the $j$ th receive antenna are denoted by $g_{T, i}^{D}(t), g_{T, i}^{M}(t), g_{R, j}^{D}(t)$, and $g_{R, j}^{M}(t)$, respectively. Then, a simplified low-pass-equivalent signal model for MIMO can be obtained as in Fig. 2 for the $j$ th receive antenna where the impulse responses of the overall direct channel $p_{i j}(t)$ and the overall mirror channel $q_{i j}(t)$ for the $i$ th transmit antenna and the $j$ th receive antenna read as

$$
\begin{aligned}
p_{i j}(t)= & g_{T, i}^{D}(t) * h_{i j}(t) * g_{R, j}^{D}(t) \\
& +\left(g_{T, i}^{M}(t)\right)^{*} * h_{i j}^{*}(t) * g_{R, j}^{M}(t) \\
q_{i j}(t)= & g_{T, i}^{M}(t) * h_{i j}(t) * g_{R, j}^{D}(t) \\
& +\left(g_{T, i}^{D}(t)\right)^{*} * h_{i j}^{*}(t) * g_{R, j}^{M}(t) .
\end{aligned}
$$

The receive filter output signal corresponding to the receive antenna $j$ and transmit signals $\left\{s_{i}(t)\right\}$ is

$$
r_{j}(t)=\sum_{i=0}^{N_{\mathrm{Tx}}-1}\left(s_{i}(t) * p_{i}(t)+s_{i}^{*}(t) * q_{i}(t)\right)+n_{j}(t)
$$

where the complex Gaussian noise $n_{j}(t)$ is given by

$$
n_{j}(t)=w(t) * g_{R, j}^{D}(t)+w^{*}(t) * g_{R, j}^{M}(t) .
$$

When MIMO channels are independent or their joint statistics are unknown, the logical approach to the estimation of the equivalent direct and mirror channels is to estimate them at each receive antenna independently. Hence, we just need to describe them for one receive antenna. In the following, we drop the receive antenna index $j$. We consider an OFDM system with a cyclic prefix $(\mathrm{CP})$ interval $\left(N_{\mathrm{CP}}\right.$ samples) longer than the maximum span ( $L$ samples) of $\left\{p_{i}(t)\right\}$ and $\left\{q_{i}(t)\right\}$.

Now, consider a low-pass-equivalent discrete-time OFDM system with $N$ subcarriers. The channels are assumed to be constant over $K$ symbols. The discrete-time transmit training signal from the $i$ th transmit antenna during the $l$ th symbol is denoted by $s_{i}^{(l)}[k]$ with the integer index $k \in\left[-N_{\mathrm{CP}}, N-1\right]$, and the $\mathrm{CP}$ samples $s_{i}^{(l)}[m]=s_{i}^{(l)}[N-m]$ for $m \in$ $\left[-N_{\mathrm{CP}},-1\right]$. Similar notations apply to the data signal $x_{i}^{(l)}[k]$. The discrete-time versions of $p_{i}(t)$ and $q_{i}(t)$ are denoted by $L \times 1$ vectors $\boldsymbol{p}_{i}$ and $\boldsymbol{q}_{i}$, respectively. The time-domain $N \times 1$ received signal vector after the $\mathrm{CP}$ removal at each receive antenna for the $l$ th OFDM symbol, denoted by $\boldsymbol{r}_{l}$, can be expressed in a general pilot-data multiplexed setup (which includes pilot only or data only symbols as special cases) as

$$
\boldsymbol{r}_{l}=\sum_{i=0}^{N_{\mathrm{Tx}}-1}\left\{\left(\boldsymbol{S}_{i}[l]+\boldsymbol{X}_{i}[l]\right) \boldsymbol{p}_{i}+\left(\boldsymbol{S}_{i}^{*}[l]+\boldsymbol{X}_{i}^{*}[l]\right) \boldsymbol{q}_{i}\right\}+\boldsymbol{n}_{l}
$$

where $(m, k)$ th element of the $N \times L$ signal matrix $\boldsymbol{S}_{i}[l]$ (or $\left.\boldsymbol{X}_{i}[l]\right)$ is $s_{i}^{(l)}[m-k]\left(\right.$ or $\left.x_{i}^{(l)}[m-k]\right)$ with $m \in[0, N-1]$ and $k \in[0, L-1]$. The received signal vector for $K$ OFDM symbols is given by

$$
\boldsymbol{r}=\boldsymbol{S p}+\boldsymbol{S}^{*} \boldsymbol{q}+\boldsymbol{X} \boldsymbol{p}+\boldsymbol{X}^{*} \boldsymbol{q}+\boldsymbol{n}
$$

where $\boldsymbol{r}=\left[\boldsymbol{r}_{0}^{T} \boldsymbol{r}_{1}^{T} \ldots \boldsymbol{r}_{K-1}^{T}\right]^{T}, \boldsymbol{p}=\left[\boldsymbol{p}_{0}^{T} \boldsymbol{p}_{1}^{T} \ldots \boldsymbol{p}_{N_{\mathrm{TX}-1}}^{T}\right]^{T}$, $\boldsymbol{q}=\left[\boldsymbol{q}_{0}^{T} \boldsymbol{q}_{1}^{T} \ldots \boldsymbol{q}_{N_{T \times-1}^{T}}^{T}\right]^{T}, \boldsymbol{n}=\left[\boldsymbol{n}_{0}^{T} \boldsymbol{n}_{1}^{T} \ldots \boldsymbol{n}_{K-1}^{T}\right]^{T}$, the $(k, i)$ th submatrice of $\boldsymbol{S}$ and $\boldsymbol{X}$ are respectively given by $\boldsymbol{S}_{i}[k]$ and $\boldsymbol{X}_{i}[k]$, with $k \in[0, K-1]$ and $i \in\left[0, N_{\mathrm{Tx}}-1\right]$. The complex Gaussian noise vectors $\left\{\boldsymbol{n}_{l}\right\}$ are given by

$$
\boldsymbol{n}_{l}=\boldsymbol{G}_{R, D} \boldsymbol{w}_{l}+\boldsymbol{G}_{R, M} \boldsymbol{w}_{l}^{*}
$$

where $\left\{\boldsymbol{w}_{l}\right\}$ are independent and identically distributed (i.i.d.) random vectors, each consisting of i.i.d. circularly-symmetric complex Gaussian random variables with the variance $\sigma_{w}^{2}$. Let $\lambda$ denote the maximum of the numbers of taps of $g_{R}^{D}[k]$ and $g_{R}^{Q}[k]$. Then, $\boldsymbol{G}_{R, D}$ and $\boldsymbol{G}_{R, M}$ are $N \times(N+\lambda)$ matrices with their first rows given by $\left[g_{R}^{D}[0], g_{R}^{D}[1], \ldots, g_{R}^{D}[\lambda], \mathbf{0}_{1 \times N-1}\right]$ and $\left[g_{R}^{M}[0], g_{R}^{M}[1], \ldots, g_{R}^{M}[\lambda], \mathbf{0}_{1 \times N-1}\right]$, respectively, and their next $k$ th rows are cyclically $k$-right-shift versions of their corresponding first rows.

Let $c_{i, l}[k]$ and $b_{i, l}[k]$, respectively, denote the pilot symbol and the data symbol on the $k$ th subcarrier of the $l$ th OFDM symbol from the $i$ th transmit antenna. Define $\boldsymbol{\Lambda}_{i, l} \triangleq$ $\operatorname{diag}\left\{c_{i, l}[0], c_{i, l}[1], \ldots, c_{i, l}[N-1]\right\}$ and $\boldsymbol{D}_{i, l} \triangleq \operatorname{diag}\left\{b_{i, l}[0]\right.$, $\left.b_{i, l}[1], \ldots, b_{i, l}[N-1]\right\}$. Let $\boldsymbol{F}_{L}$ denote the first $L$ columns of the $N \times N$ unitary discrete Fourier transform (DFT) matrix $\boldsymbol{F}$. Then the time domain signal matrices can be given as

$$
\boldsymbol{S}_{i}[l]=\sqrt{N} \boldsymbol{F}^{H} \boldsymbol{\Lambda}_{i, l} \boldsymbol{F}_{L}, \quad \boldsymbol{X}_{i}[l]=\sqrt{N} \boldsymbol{F}^{H} \boldsymbol{D}_{i, l} \boldsymbol{F}_{L} .
$$

Define $\boldsymbol{P} \triangleq \boldsymbol{F} \boldsymbol{F}^{T}$. Then, we have $\boldsymbol{P}=\boldsymbol{P}^{T}, \boldsymbol{P} \boldsymbol{P}^{T}=\boldsymbol{I}_{N}$, $\boldsymbol{P} \boldsymbol{F}_{L}^{*}=\boldsymbol{F}_{L}$, and

$$
\begin{aligned}
\tilde{\boldsymbol{\Lambda}}_{i, l} & \triangleq \boldsymbol{P} \boldsymbol{\Lambda}_{i, l}^{H} \boldsymbol{P} \\
& =\operatorname{diag}\left\{c_{i, l}^{*}[0], c_{i, l}^{*}[N-1], c_{i, l}^{*}[N-2], \ldots, c_{i, l}^{*}[2], c_{i, l}^{*}[1]\right\} \\
\tilde{\boldsymbol{D}}_{i, l} & \triangleq \boldsymbol{P} \boldsymbol{D}_{i, l}^{H} \boldsymbol{P} \\
& =\operatorname{diag}\left\{b_{i, l}^{*}[0], b_{i, l}^{*}[N-1], b_{i, l}^{*}[N-2], \ldots, b_{i, l}^{*}[2], b_{i, l}^{*}[1]\right\} .
\end{aligned}
$$

From the above and the DFT of (9), the received symbol 
$R_{l}[k]$ on the $k$ th subcarrier of the $l$ th OFDM symbol reads as

$$
\begin{aligned}
R_{l}[k]=\sum_{i=0}^{N_{\mathrm{Tx}}-1} & \left\{\left(c_{i, l}[k]+b_{i, l}[k]\right) P_{i}[k]\right. \\
& \left.+\left(c_{i, l}^{*}[-k]+b_{i, l}^{*}[-k]\right) Q_{i}[k]\right\}
\end{aligned}
$$

where $P_{i}[k]$ and $Q_{i}[k]$ are the $k$ th elements of $\sqrt{N} \boldsymbol{F}_{L} \boldsymbol{p}_{i}$ and $\sqrt{N} \boldsymbol{F}_{L} \boldsymbol{q}_{i}$, respectively. Clearly, the I/Q imbalance introduces to the received $k$ th subcarrier symbol an interference from the mirror tone (i.e., index $-k$ ) with a FD coefficient of $Q_{i}[k]$. This interference, if not properly taken into account, can cause a significant performance degradation.

\section{MIMO OFDM PILOT DESIGN CRITERIA}

For a coherent detection, the direct channel $\boldsymbol{p}$ and the mirror channel $\boldsymbol{q}$ need to be estimated at the receiver. In practical systems, the statistics of the channel and the transceiver imperfections are unknown and they can be non-stationary as well. This leads to the practical choice of least-squares type channel estimators as considered in this paper. The estimates of the direct and mirror CIR vectors are given by

$$
\begin{aligned}
& \hat{\boldsymbol{p}}=\left(\boldsymbol{S}^{H} \boldsymbol{S}\right)^{-1} \boldsymbol{S}^{H} \boldsymbol{r} \\
& \hat{\boldsymbol{q}}=\left(\boldsymbol{S}^{T} \boldsymbol{S}^{*}\right)^{-1} \boldsymbol{S}^{T} \boldsymbol{r} .
\end{aligned}
$$

Our pilot designs (and the optimality criterion) will be based on minimizing the channel estimation mean-square error (MSE). Substituting (10) into (16) and (17) gives

$$
\begin{aligned}
\hat{\boldsymbol{p}}= & \left(\boldsymbol{S}^{H} \boldsymbol{S}\right)^{-1} \boldsymbol{S}^{H} \boldsymbol{S} \boldsymbol{p}+\left(\boldsymbol{S}^{H} \boldsymbol{S}\right)^{-1} \boldsymbol{S}^{H} \boldsymbol{S}^{*} \boldsymbol{q} \\
& +\left(\boldsymbol{S}^{H} \boldsymbol{S}\right)^{-1} \boldsymbol{S}^{H} \boldsymbol{X} \boldsymbol{p}+\left(\boldsymbol{S}^{H} \boldsymbol{S}\right)^{-1} \boldsymbol{S}^{H} \boldsymbol{X}^{*} \boldsymbol{q} \\
& +\left(\boldsymbol{S}^{H} \boldsymbol{S}\right)^{-1} \boldsymbol{S}^{H} \boldsymbol{n} \\
\hat{\boldsymbol{q}}= & \left(\boldsymbol{S}^{T} \boldsymbol{S}^{*}\right)^{-1} \boldsymbol{S}^{T} \boldsymbol{S}^{*} \boldsymbol{q}+\left(\boldsymbol{S}^{T} \boldsymbol{S}^{*}\right)^{-1} \boldsymbol{S}^{T} \boldsymbol{S} \boldsymbol{p} \\
& +\left(\boldsymbol{S}^{T} \boldsymbol{S}^{*}\right)^{-1} \boldsymbol{S}^{T} \boldsymbol{X} \boldsymbol{p}+\left(\boldsymbol{S}^{T} \boldsymbol{S}^{*}\right)^{-1} \boldsymbol{S}^{T} \boldsymbol{X}^{*} \boldsymbol{q} \\
& +\left(\boldsymbol{S}^{T} \boldsymbol{S}^{*}\right)^{-1} \boldsymbol{S}^{T} \boldsymbol{n} .
\end{aligned}
$$

All five terms in each of the above equations can affect the MSE. The second to the fourth terms are interferences and their contributions to the MSE is minimized if they become zeros (no interferences). The first term needs to yield the parameter under estimation so that its contribution to the MSE is minimized (zero). The last term is due to the noise and when its covariance matrix is unknown (for the most general case), minimization of its contribution to MSE is impractical. However, in practice, the noise term is almost white as will be explained below. Under this condition, the first and the last terms are exactly the same as the system in [11] and hence, the MSE-minimizing condition (due to the first and the last terms) is the same as that in [11]. All of the above mentioned (practically feasible) MSE-minimizing conditions can be elaborated as follows:

1) Estimation Identifiability Condition: The identifiability of $\boldsymbol{p}$ and $\boldsymbol{q}$ estimation requires that $\boldsymbol{S}^{H} \boldsymbol{S}$ is of full-rank.
2) Zero Cross Channel Interference Condition: The MSE due to the second term (cross channel interference) in (18) and (19) is removed when $\boldsymbol{S}^{H} \boldsymbol{S}^{*}=\mathbf{0}_{L N_{\mathrm{Tx}}}$.

3) Zero Data Interference Condition: The random data interference is completely suppressed when $\boldsymbol{S}^{H} \boldsymbol{X}=$ $\mathbf{0}_{L N_{\mathrm{Tx}}}$ and $\boldsymbol{S}^{H} \boldsymbol{X}^{*}=\mathbf{0}_{L N_{\mathrm{Tx}}}$.

4) White Noise Optimality Condition: When the equivalent receive-filter is a square-root Nyquist filter, the MSE due to the noise is minimized when $\boldsymbol{S}^{H} \boldsymbol{S}=E_{K} \boldsymbol{I}_{L N_{\mathrm{Tx}}}$ where $E_{K}$ is the training signal energy from a transmit antenna over $K$ symbols (excluding CPs). The FI receiver I/Q imbalance with a square-root raised cosine receive filter represents this scenario.

5) For the scenario with FD receiver I/Q imbalance, the noise covariance matrix is unknown a priori, and hence it is infeasible to develop optimal pilot designs. However, the frequency selectivity of the receiver I/Q imbalance is typically very small (and the noise would be almost white) since the amplifiers/filters are designed to have a frequency-flat response within the transmission band (see also [4], [21], [27], [28]). A practical approach in this case is to assume frequency-flat receiver I/Q imbalance $^{1}$ in the pilot designs which leads to the requirement of $\boldsymbol{S}^{H} \boldsymbol{S}=E_{K} \boldsymbol{I}_{L N_{\mathrm{Tx}}}$.

When the identifiability and zero data interference conditions are met, the MSEs of $\boldsymbol{p}$ and $\boldsymbol{q}$ estimation become

$$
\begin{aligned}
\operatorname{MSE}_{\boldsymbol{p}}=\operatorname{Tr} & {\left[\left(\boldsymbol{S}^{H} \boldsymbol{S}\right)^{-1} \boldsymbol{S}^{H} \boldsymbol{S}^{*} E\left[\boldsymbol{q} \boldsymbol{q}^{H}\right] \boldsymbol{S}^{T} \boldsymbol{S}\left(\boldsymbol{S}^{H} \boldsymbol{S}\right)^{-1}\right.} \\
+ & \left.\left(\boldsymbol{S}^{H} \boldsymbol{S}\right)^{-1} \boldsymbol{S}^{H} \boldsymbol{C}_{\boldsymbol{n}} \boldsymbol{S}\left(\boldsymbol{S}^{H} \boldsymbol{S}\right)^{-1}\right] \\
\mathrm{MSE}_{\boldsymbol{q}}=\operatorname{Tr} & {\left[\left(\boldsymbol{S}^{T} \boldsymbol{S}^{*}\right)^{-1} \boldsymbol{S}^{T} \boldsymbol{S} E\left[\boldsymbol{p} \boldsymbol{p}^{H}\right] \boldsymbol{S}^{H} \boldsymbol{S}^{*}\left(\boldsymbol{S}^{T} \boldsymbol{S}^{*}\right)^{-1}\right.} \\
+ & \left.\left(\boldsymbol{S}^{T} \boldsymbol{S}^{*}\right)^{-1} \boldsymbol{S}^{T} \boldsymbol{C}_{\boldsymbol{n}} \boldsymbol{S}^{*}\left(\boldsymbol{S}^{T} \boldsymbol{S}^{*}\right)^{-1}\right]
\end{aligned}
$$

where the noise covariance matrix $C_{n}$ is given by

$$
\boldsymbol{C}_{\boldsymbol{n}}=\sigma_{w}^{2}\left(\boldsymbol{G}_{R, D} \boldsymbol{G}_{R, D}^{H}+\boldsymbol{G}_{R, M} \boldsymbol{G}_{R, M}^{H}\right) \otimes \boldsymbol{I}_{K} .
$$

If the zero cross channel interference condition is also met, the above MSE expressions become

$$
\operatorname{MSE}_{\boldsymbol{p}}=\mathrm{MSE}_{\boldsymbol{q}}=\operatorname{Tr}\left[\left(\boldsymbol{S}^{H} \boldsymbol{S}\right)^{-1} \boldsymbol{S}^{H} \boldsymbol{C}_{\boldsymbol{n}} \boldsymbol{S}\left(\boldsymbol{S}^{H} \boldsymbol{S}\right)^{-1}\right]
$$

Additionally, if the demodulator output noise samples are white (i.e., $\boldsymbol{C}_{\boldsymbol{n}}=\sigma_{n}^{2} \boldsymbol{I}$ ), the MSE expressions simplify to $\mathrm{MSE}_{\boldsymbol{p}}=\mathrm{MSE}_{\boldsymbol{q}}=\sigma_{n}^{2} \operatorname{Tr}\left[\left(\boldsymbol{S}^{H} \boldsymbol{S}\right)^{-1}\right]$.

The MIMO OFDM pilot design criterion satisfying the estimation identifiability, zero data interference condition, zero cross channel interference, and white noise optimality reads as

$$
\left.\begin{array}{l}
\left(\boldsymbol{S}^{H} \boldsymbol{S}\right)=E_{K} \boldsymbol{I}_{N_{\mathrm{Tx}} L} \quad \& \quad\left(\boldsymbol{S}^{H} \boldsymbol{S}^{*}\right)=\mathbf{0}_{N_{\mathrm{Tx}} L} \\
\boldsymbol{S}^{H} \boldsymbol{X}=\mathbf{0}_{N_{\mathrm{Tx}} L} \quad \& \quad \boldsymbol{S}^{H} \boldsymbol{X}^{*}=\mathbf{0}_{N_{\mathrm{Tx}} L}
\end{array}\right\}
$$

\footnotetext{
${ }^{1}$ The transmitter I/Q imbalances need not be frequency-flat since they do not affect the receiver noise statistics.
} 
The above criterion can be detailed as

$$
\begin{aligned}
& \text { Condition } 1: \sum_{l=0}^{K-1} \boldsymbol{S}_{i}^{H}[l] \boldsymbol{X}_{k}[l]=\mathbf{0}_{L}, \forall i, k \\
& \text { Condition 2: } \sum_{l=0}^{K-1} \boldsymbol{S}_{i}^{H}[l] \boldsymbol{X}_{k}^{*}[l]=\mathbf{0}_{L}, \forall i, k \\
& \text { Condition } 3: \sum_{l=0}^{K-1} \boldsymbol{S}_{i}^{H}[l] \boldsymbol{S}_{i}[l]=E_{K} \boldsymbol{I}_{L}, \forall i \\
& \text { Condition } 4: \sum_{l=0}^{K-1} \boldsymbol{S}_{i}^{H}[l] \boldsymbol{S}_{k}[l]=\mathbf{0}_{L}, \forall i \neq k \\
& \text { Condition } 5: \sum_{l=0}^{K-1} \boldsymbol{S}_{i}^{H}[l] \boldsymbol{S}_{k}^{*}[l]=\mathbf{0}_{L}, \forall i, k .
\end{aligned}
$$

The corresponding MSE becomes

$$
\begin{aligned}
\mathrm{MSE}_{\boldsymbol{p}}=\mathrm{MSE}_{\boldsymbol{q}} & =\frac{\sigma_{w}^{2}}{E_{K}^{2}} \operatorname{Tr}\left[\left(\boldsymbol { S } ^ { H } \left(\left(\boldsymbol{G}_{R, D} \boldsymbol{G}_{R, D}^{H}\right.\right.\right.\right. \\
& \left.\left.\left.\left.+\boldsymbol{G}_{R, M} \boldsymbol{G}_{R, M}^{H}\right) \otimes \boldsymbol{I}_{K}\right) \boldsymbol{S}\right)^{-1}\right] .
\end{aligned}
$$

Following [26], we can also have the Cramer-Rao lower bound (CRB) for the estimation of $\left[\boldsymbol{p}^{T}, \boldsymbol{q}^{T}\right]^{T}$ (i.e., a theoretical lower bound for $\mathrm{MSE}_{\boldsymbol{p}}+\mathrm{MSE}_{\boldsymbol{q}}$ ) as

$$
\mathrm{CRB}=\operatorname{Tr}\left[\left\{\left(\left[\boldsymbol{S}, \boldsymbol{S}^{*}\right]^{H} \boldsymbol{C}_{\boldsymbol{n}}^{-1}\left[\boldsymbol{S}, \boldsymbol{S}^{*}\right]\right)^{-1}\right\}\right],
$$

which will be used as a benchmark for the performance evaluation of the proposed pilot designs.

\section{MIMO OFDM PILOT DESIGNS}

We will first investigate what characteristics of pilots will satisfy each of the above conditions, separately. Using (12), (13) and (14), we obtain

$$
\begin{aligned}
\boldsymbol{S}_{i}^{H}[l] \boldsymbol{X}_{k}[l] & =N \boldsymbol{F}_{L}^{H} \boldsymbol{\Lambda}_{i, l}^{H} \boldsymbol{D}_{m, l} \boldsymbol{F}_{L} \\
\boldsymbol{S}_{i}^{H}[l] \boldsymbol{X}_{k}^{*}[l] & =N \boldsymbol{F}_{L}^{H} \boldsymbol{\Lambda}_{i, l}^{H} \tilde{\boldsymbol{D}}_{m, l}^{H} \boldsymbol{F}_{L} \\
\boldsymbol{S}_{i}^{H}[l] \boldsymbol{S}_{k}[l] & =N \boldsymbol{F}_{L}^{H} \boldsymbol{\Lambda}_{i, l}^{H} \boldsymbol{\Lambda}_{m, l} \boldsymbol{F}_{L} \\
\boldsymbol{S}_{i}^{H}[l] \boldsymbol{S}_{k}^{*}[l] & =N \boldsymbol{F}_{L}^{H} \boldsymbol{\Lambda}_{i, l}^{H} \tilde{\Lambda}_{m, l}^{H} \boldsymbol{F}_{L} .
\end{aligned}
$$

As data are random, using (32) and (33), we can conclude that Condition- 1 and 2 require

$$
\boldsymbol{\Lambda}_{i, l}^{H} \boldsymbol{D}_{m, l}=\mathbf{0}_{N} \quad \& \quad \boldsymbol{\Lambda}_{i, l}^{H} \tilde{\boldsymbol{D}}_{m, l}^{H}=\mathbf{0}_{N} \quad \forall i, m, l .
$$

In other words, at each OFDM symbol, the pilot tone index set for all antennas and the data tone index set for all antennas need to be disjoint and each set is composed of pairs of mirror tones only. The indexes of a mirror pair is given by $(k,-k)$ for $k=0,1, \ldots, N / 2$. Note that $k=0$ is a self-mirror tone and so is $k=N / 2$. Define $\mathcal{J}_{l} \triangleq \cup_{i=0}^{N_{\mathrm{Tx}}-1} \mathcal{J}_{l, i}$ and $\mathcal{I}_{l} \triangleq \cup_{i=0}^{N_{\mathrm{Tx}}-1} \mathcal{I}_{l, i}$ where $\mathcal{J}_{l, i}$ and $\mathcal{I}_{l, i}$ denote the pilot (including null pilot) index set and the data index set of $i$ th antenna at $l$ th OFDM symbol, respectively. Note that $\mathcal{J}_{l, i}$ can be separated into non-zero pilot tone index set $\mathcal{J}_{l, i}^{\text {pilot }}$ and null pilot tone index set $\mathcal{J}_{l, i}^{\text {null }}$. Then, Condition- 1 and 2 require that if $k \in \mathcal{J}_{l}$ and $m \in \mathcal{I}_{l}$, then $(-k)_{N} \in \mathcal{J}_{l},(-m)_{N} \in \mathcal{I}_{l}$, and $k \neq m$.
Next, by using (12), Condition-3 becomes

$$
\sum_{l=0}^{K-1} \boldsymbol{F}_{L}^{H} \Lambda_{i, l}^{H} \Lambda_{i, l} \boldsymbol{F}_{L}=E_{K} \boldsymbol{I}_{L}, \forall i
$$

or equivalently, for $d \in\{-L+1,-L+2, \ldots, L-1\}$,

$$
\sum_{k=0}^{N-1}\left(\sum_{l=0}^{K-1}\left|c_{i, l}[k]\right|^{2}\right) e^{j 2 \pi d k / N}=E_{K} \delta[d] .
$$

For a typical FFT size $N$ which is a power of 2 , define $L_{0}=$ $2^{\left\lceil\log _{2}(\mathrm{~L})\right\rceil}, L_{i}=2^{i} L_{0}$, and $M_{i}=N / L_{i}$. Then, Condition-3 is satisfied when

$$
\sum_{l=0}^{K-1}\left|c_{i, l}[k]\right|^{2}=\sum_{m=0}^{\log _{2}\left(M_{0}\right)-1} \sum_{l=0}^{M_{m}-1} \sum_{n=0}^{L_{m}-1} a_{m, l} \delta\left[k-n M_{m}-\tau_{m, l}\right],
$$

where $\left\{a_{m, l}\right\}$ take on real non-negative values and satisfy

$$
\sum_{m=0}^{\log _{2}\left(M_{0}\right)} \sum_{l=0}^{-1} a_{m, l}=E_{K}, a_{m, l} \geq 0, \tau_{m, l} \in\left[0, M_{m}-1\right] .
$$

Similarly, Condition-4 becomes

$$
\sum_{l=0}^{K-1} \boldsymbol{F}_{L}^{H} \boldsymbol{\Lambda}_{i, l}^{H} \boldsymbol{\Lambda}_{m, l} \boldsymbol{F}_{L}=\mathbf{0}_{L}, \quad \forall i \neq m
$$

or equivalently, for $d \in\{-L+1,-L+2, \ldots, L-1\}$,

$$
\sum_{k=0}^{N-1} \sum_{l=0}^{K-1} c_{i, l}^{*}[k] c_{m, l}[k] e^{j 2 \pi d k / N}=0, \forall i \neq m .
$$

With the definitions of $G_{i, m}[l, k] \triangleq c_{i, l}^{*}[k] c_{m, l}[k]$ and $\left\{g_{i, m}[l, n]\right\}$ being the inverse DFT of $\left\{G_{i, m}[l, k]\right\}$, a sufficient condition for satisfying Condition-4 is either of the two following conditions:

$$
\begin{aligned}
& \sum_{l=0}^{K-1} G_{i, m}[l, k]=0, \quad \forall i \neq m, \forall k \\
& g_{i, m}[l, k]=0, \\
& k=0,1, \ldots, L-1, N-L+1, \ldots, N-1, \quad \forall i \neq m .
\end{aligned}
$$

Condition-5 can be expressed as

$$
\sum_{l=0}^{K-1} \boldsymbol{F}_{L}^{H} \boldsymbol{\Lambda}_{i, l}^{H} \boldsymbol{F} \boldsymbol{F}^{T} \boldsymbol{\Lambda}_{m, l}^{H} \boldsymbol{F}_{L}^{*}=\mathbf{0}_{L}, \quad \forall i, m .
$$

Then, Condition- 5 becomes, for all $i$ and $m$,

$$
\sum_{l=0}^{K-1} \boldsymbol{F}_{L}^{H} \boldsymbol{\Lambda}_{i, l}^{H} \tilde{\boldsymbol{\Lambda}}_{m, l}^{H} \boldsymbol{F}_{L}=\boldsymbol{F}_{L}^{H}\left(\sum_{l=0}^{K-1} \boldsymbol{\Lambda}_{i, l}^{H} \tilde{\boldsymbol{\Lambda}}_{m, l}^{H}\right) \boldsymbol{F}_{L}=\mathbf{0}_{L},
$$

or equivalently, for $d \in\{-L+1,-L+2, \ldots, L-1\}$,

$$
\sum_{k=0}^{N-1}\left(\sum_{l=0}^{K-1} c_{i, l}^{*}[k] c_{m, l}^{*}[N-k]\right) e^{j 2 \pi d k / N}=0, \forall i, m .
$$


$\left\{\left(\sum_{l=0}^{K-1} c_{i, l}^{*}[k] c_{m, l}^{*}[N-k]\right): k=0, \ldots, N-1\right\} \quad$ be in the null space of the columns of $\boldsymbol{F}_{L}$ and $\boldsymbol{F}_{L}^{H}, \forall i, m, k$, as

$$
\begin{aligned}
& \sum_{l=0}^{K-1} c_{i, l}^{*}[k] c_{m, l}^{*}[N-k]=0 \\
& \sum_{l=0}^{K-1} c_{i, l}^{*}[k] c_{m, l}^{*}[N-k]=e^{j 2 \pi \bar{d} k / N}, \bar{d} \in\{L, \ldots, N-L\},
\end{aligned}
$$

or, for $d=-(L-1), \ldots,(L-1)$,

$$
\sum_{k=0}^{N-1} c_{i, l}[k] c_{m, l}[N-k] e^{j 2 \pi d k / N}=0, \forall i, m, l .
$$

By considering $i=m$ in (50), one can easily find that $2 L_{0}$ pilot tones (may include null pilots) are necessary for one transmit antenna.

Combining all of the above pilot characteristics, we present several pilot designs which satisfy the five design criteria. These designs will be denoted by two terms - how pilots of different antennas are decoupled and how mirror tone interferences are suppressed. The same pilot signal energy over $K$ symbols for each antenna as required in Condition3 will not be explicitly mentioned in the following designs. Without loss of generality, we will present unit-amplitude pilot symbols. The existing MIMO OFDM pilot designs from [11], namely time division multiplexing (TDM), code division multiplexing across time domain (CDM-T), CDM across frequency domain (CDM-F), frequency division multiplexing (FDM), and time and frequency division multiplexing (TFDM), will be extensively used in our designs. Due to space limitation, details of these existing designs are referred to [11]. How our pilot designs satisfy the five design criteria will be briefly mentioned for the first design, but it should be obvious and hence will be skipped for the other designs. Examples of our pilot designs are provided in Tables I and II for illustration of the concepts where parameters are chosen for the convenience of the presentation. In some designs, several options are presented by separating them with dashed lines.

\section{A. [TDM; TD / C-F] Design}

In this design, pilots of different antennas are decoupled by TDM design while mirror tone interferences are suppressed by means of time disjointness (TD) or code design in frequency domain (C-F). The pilot and data index sets for the $i$ th transmit antenna at the $l$ th OFDM symbol are given by

$$
\begin{aligned}
\mathcal{J}_{l, i} & = \begin{cases}\left\{0, M_{0} / 2, M_{0}, \ldots, N-M_{0} / 2\right\}, & \text { if } i=l \\
\emptyset, & \text { else }\end{cases} \\
\mathcal{I}_{l} & =\{0,1,2, \ldots, N-1\} \backslash \mathcal{J}_{l}
\end{aligned}
$$

where TDM pilot assignment for different antennas can be easily noticed. Due to TDM, there is no mirror tone interference across antennas (i.e., mirror tone interference suppression through TD). For each antenna, the pilots are given by

$$
\begin{aligned}
& c_{i, i}[0]= \pm c_{i, i}[N / 2]= \pm 1 \text { or } \pm j \\
& c_{i, i}[k]=e^{j \phi_{i, k}}, \text { arbitrary } \phi_{i, k}, \\
& \quad k \in\left\{M_{0} / 2, M_{0}, \ldots,\left(N-M_{0}\right) / 2\right\} \\
& c_{i, i}[k]=(-1)^{(2 k-N) / M_{0}} c_{i, i}^{2}[0] c_{i, i}^{*}[-k], \\
& \quad k \in\left\{\left(N+M_{0}\right) / 2, N / 2+M_{0}, \ldots, N-M_{0} / 2\right\} \\
& c_{i, i}[k]=0, k \notin \mathcal{J}_{i, i} .
\end{aligned}
$$

For example, $\left\{c_{i, i}[k]: k \in \mathcal{J}_{i, i}\right\}$ can be either $\left\{ \pm 1, a_{1}, a_{2}\right.$, $\left.\ldots, a_{L_{0}-1}, \quad \pm 1,-a_{L_{0}-1}^{*}, \quad a_{L_{0}-2}^{*}, \quad-a_{L_{0}-3}^{*}, \quad \ldots, a_{2}^{*},-a_{1}^{*}\right\}$ or $\left\{ \pm j, a_{1}, a_{2}, \ldots, a_{L_{0}-1}, \quad \pm j, a_{L_{0}-1}^{*}, \quad-a_{L_{0}-2}^{*}, a_{L_{0}-3}^{*}\right.$, $\left.\ldots,-a_{2}^{*}, a_{1}^{*}\right\}$ where $\left\{a_{k}\right\}$ are arbitrary unit amplitude symbols. The above pilot codes across the frequency domain are designed such that the mirror interference becomes zero, i.e., to satisfy (50) for $i=m$. Note that (51) and (52) guarantee Conditions 1 and 2, while TDM fulfills Conditions 3 and 4. TDM and C-F guarantee Condition 5. This design requires $2 L_{0}$ pilot tones in each symbol and a total of $K=N_{\text {Tx }}$ symbols. $L_{m}$ and $M_{m}$ with $m>0$ can be used instead of $L_{0}$ and $M_{0}$, but they cost more overhead.

\section{B. [CDM-T; C-T] Design Without Self-Mirror Tones}

Pilots of different antennas are decoupled by CDM-T design. Pilot mirror tone interferences are suppressed by code design across time domain (C-T). The pilot and data index sets are given by

$$
\begin{aligned}
\mathcal{J}_{l, i} & =\left\{\frac{M_{0}}{2}, \frac{3 M_{0}}{2}, \frac{5 M_{0}}{2}, \ldots, N-\frac{M_{0}}{2}\right\}, \quad \forall i, l \\
\mathcal{I}_{l} & =\{0,1,2, \ldots, N-1\} \backslash \mathcal{J}_{l}
\end{aligned}
$$

where self-mirror tones (i.e., index 0 and $N / 2$ ) are excluded from the pilot index set. Define

$$
\begin{gathered}
\boldsymbol{c}_{i}[k] \triangleq\left[c_{i, 0}[k], c_{i, 1}[k], \ldots, c_{i, K-1}[k]\right]^{T} \\
{\left[\boldsymbol{v}_{m}\right]_{k} \triangleq e^{j 2 \pi m k / K}, m, k \in\{0,1, \ldots, K-1\},} \\
K \geq 2\left(N_{\mathrm{Tx}}+n\right), n \in\{0,1,2, \ldots\} .
\end{gathered}
$$

For $k \in\left\{M_{0} / 2,3 M_{0} / 2, \ldots, N / 2-M_{0} / 2\right\}$, the pilots are given as

$$
\begin{aligned}
\boldsymbol{c}_{0}[k] & =e^{j \phi_{0, k}}, k \in \mathcal{J}_{0}^{\text {pilot }} \\
\boldsymbol{c}_{0}[-k] & =e^{j \phi_{0,-k}} \operatorname{diag}\left\{\boldsymbol{v}_{1}\right\} \boldsymbol{c}_{0}^{*}[k] \\
\boldsymbol{c}_{i}[k] & =e^{j \phi_{i, k}} \operatorname{diag}\left\{\boldsymbol{v}_{2 i}\right\} \boldsymbol{c}_{0}[k], i=1, \ldots, N_{\mathrm{Tx}}-1 \\
\boldsymbol{c}_{i}[-k] & =e^{j \phi_{i,-k}} \operatorname{diag}\left\{\boldsymbol{v}_{2 i+1}\right\} \boldsymbol{c}_{0}^{*}[k], i=1, \ldots, N_{\mathrm{Tx}}-1
\end{aligned}
$$

where $\left\{\phi_{i, \pm k}\right\}$ are arbitrary. In the above design, for $k \in$ $\left\{M_{0} / 2,3 M_{0} / 2, \ldots, N / 2-M_{0} / 2\right\},\left\{\boldsymbol{c}_{i}[k]\right\}$ for different antenna $i \in\left\{0,1, \ldots, N_{\mathrm{Tx}}-1\right\}$ are constructed using $\operatorname{diag}\left\{\boldsymbol{v}_{2 i}\right\}$ while $\left\{\boldsymbol{c}_{i}[-k]\right\}$ are based on $\operatorname{diag}\left\{\boldsymbol{v}_{2 i+1}\right\}$. Note that any permutation of $\left\{v_{i}: i=1, \ldots, 2 N_{\mathrm{Tx}}-1\right\}$ can be associated to $\left\{\boldsymbol{c}_{i}[k]\right\}$ in the above equations. Overhead consideration will set $K=2 N_{\mathrm{Tx}}$. Although $M_{i}$ can be used in place of $M_{0}$, it is not desirable due to larger overhead. 
TABLE I

EXAMPLES OF Proposed Pilot Designs

\begin{tabular}{|c|c|c|c|}
\hline Design & $\begin{array}{l}\text { antenna } \\
\text { index } i\end{array}$ & $\begin{array}{l}\text { symbol } \\
\text { index } l\end{array}$ & Pilots \\
\hline $\begin{array}{c}\text { [TDM; TD / C-F }] \\
N=16, L_{0}=4, M_{0}=4 \\
K=2, N_{\mathrm{Tx}}=2\end{array}$ & $\begin{array}{l}0 \\
1 \\
\end{array}$ & $\begin{array}{l}0 \\
1 \\
\end{array}$ & $\begin{array}{c}\mathcal{J}_{l, i}=\{0,2,4,6,8,10,12,14\} \\
c_{0,0}\left[k \in \mathcal{J}_{l, i}\right]=\left\{j, a_{1}, a_{2}, a_{3}, j, a_{3}^{*},-a_{2}^{*}, a_{1}^{*}\right\} \\
c_{1,1}\left[k \in \mathcal{J}_{l, i}\right]=\left\{j, a_{4}, a_{5}, a_{6},-j, a_{6}^{*},-a_{5}^{*}, a_{4}^{*}\right\}\end{array}$ \\
\hline $\begin{array}{c}\text { [TDM; TD / C-F }] \\
N=16, L_{0}=4, M_{0}=4 \\
K=2, N_{\mathrm{Tx}}=2\end{array}$ & $\begin{array}{l}0 \\
1\end{array}$ & $\begin{array}{l}0 \\
1\end{array}$ & $\begin{array}{c}\mathcal{J}_{l, i}=\{0,2,4,6,8,10,12,14\} \\
c_{0,0}\left[k \in \mathcal{J}_{l, i}\right]=\left\{1, a_{1}, a_{2}, a_{3}, 1,-a_{3}^{*}, a_{2}^{*},-a_{1}^{*}\right\} \\
c_{1,1}\left[k \in \mathcal{J}_{l, i}\right]=\left\{-1, a_{4}, a_{5}, a_{6}, 1,-a_{6}^{*}, a_{5}^{*},-a_{4}^{*}\right\}\end{array}$ \\
\hline $\begin{array}{c}{[\text { CDM-T; C-T }]} \\
\text { without self-mirror } \\
N=16, L_{0}=4, M_{0}=4 \\
K=4, N_{\mathrm{Tx}}=2\end{array}$ & $\begin{array}{l}0 \\
0 \\
0 \\
0 \\
1 \\
1 \\
1 \\
1\end{array}$ & $\begin{array}{l}0 \\
1 \\
2 \\
3 \\
0 \\
1 \\
2 \\
3 \\
\end{array}$ & $\begin{array}{c}\mathcal{J}_{l, i}=\{2,6,10,14\} \\
c_{0,0}\left[k \in \mathcal{J}_{l, i}\right]=\left\{a_{1}, a_{5}, a_{5}^{*}, a_{1}^{*}\right\} \\
c_{1,0}\left[k \in \mathcal{J}_{l, i}\right]=\left\{a_{2}, a_{6}, a_{6}^{*} e^{j \pi / 2}, a_{2}^{*} e^{j \pi / 2}\right\} \\
c_{2,0}\left[k \in \mathcal{J}_{l, i}\right]=\left\{a_{3}, a_{7},-a_{7}^{*},-a_{3}^{*}\right\} \\
c_{3,0}\left[k \in \mathcal{J}_{l, i}\right]=\left\{a_{4}, a_{8}, a_{8}^{*} e^{-j \pi / 2}, a_{4}^{*} e^{-j \pi / 2}\right\} \\
c_{0,1}\left[k \in \mathcal{J}_{l, i}\right]=\left\{a_{1}, a_{5}, a_{5}^{*}, a_{1}^{*}\right\} \\
c_{1,1}\left[k \in \mathcal{J}_{l, i}\right]=\left\{-a_{2},-a_{6}, a_{6}^{*} e^{j \pi / 2}, a_{2}^{*} e^{j \pi / 2}\right\} \\
c_{2,1}\left[k \in \mathcal{J}_{l, i}\right]=\left\{a_{3}, a_{7},-a_{7}^{*},-a_{3}^{*}\right\} \\
c_{3,1}\left[k \in \mathcal{J}_{l, i}\right]=\left\{-a_{4},-a_{8}, a_{8}^{*} e^{-j \pi / 2}, a_{4}^{*} e^{-j \pi / 2}\right\}\end{array}$ \\
\hline $\begin{array}{c}\text { [CDM-T; C-T }] \\
\text { with self-mirror } \\
N=16, L_{0}=4, M_{0}=4 \\
K=4, N_{\mathrm{Tx}}=2\end{array}$ & $\begin{array}{l}0 \\
0 \\
0 \\
0 \\
1 \\
1 \\
1 \\
1 \\
\end{array}$ & $\begin{array}{l}0 \\
1 \\
2 \\
3 \\
0 \\
1 \\
2 \\
3 \\
\end{array}$ & $\begin{array}{c}\mathcal{J}_{l, i}=\{0,4,8,12\} \\
c_{0,0}\left[k \in \mathcal{J}_{l, i}\right]=\left\{1, a_{1}, 1, a_{1}^{*}\right\} \\
c_{1,0}\left[k \in \mathcal{J}_{l, i}\right]=\left\{e^{j \pi / 4}, a_{2}, e^{j \pi / 4}, a_{2}^{*} e^{j \pi / 2}\right\} \\
c_{2,0}\left[k \in \mathcal{J}_{l, i}\right]=\left\{e^{j \pi / 2}, a_{3}, e^{j \pi / 2},-a_{3}^{*}\right\} \\
c_{3,0}\left[k \in \mathcal{J}_{l, i}\right]=\left\{e^{-j \pi / 4}, a_{4}, e^{-j \pi / 4}, a_{4}^{*} e^{-j \pi / 2}\right\} \\
c_{0,1}\left[k \in \mathcal{J}_{l, i}\right]=\left\{1, a_{1}, 1, a_{1}^{*}\right\} \\
c_{1,1}\left[k \in \mathcal{J}_{l, i}\right]=\left\{e^{j \pi / 4}, a_{2}, e^{j \pi / 4}, a_{2}^{*} e^{j \pi / 2}\right\} \\
c_{2,1}\left[k \in \mathcal{J}_{l, i}\right]=\left\{e^{j \pi / 2}, a_{3}, e^{j \pi / 2},-a_{3}^{*}\right\} \\
c_{3,1}\left[k \in \mathcal{J}_{l, i}\right]=\left\{e^{-j \pi / 4}, a_{4}, e^{-j \pi / 4}, a_{4}^{*} e^{-j \pi / 2}\right\} \\
\end{array}$ \\
\hline $\begin{array}{c}{[\text { CDM-F; Null }]} \\
N=16, L_{0}=2, M_{0}=8 \\
K=1, N_{\mathrm{Tx}}=2\end{array}$ & $\begin{array}{l}0 \\
1\end{array}$ & $\begin{array}{l}0 \\
0 \\
\end{array}$ & $\begin{array}{c}\mathcal{J}_{i}^{\text {pilot }}=\{1,2,9,10\} \\
\mathcal{J}_{i}^{\text {null }}=\{6,7,14,15\} \\
c_{0}\left[k \in \mathcal{J}_{i}^{\text {pilot }}\right]=\left\{a_{1}, a_{2}, a_{3}, a_{4}\right\} \\
c_{1}\left[k \in \mathcal{J}_{i}^{\text {pilot }}\right]=\left\{a_{1},-a_{2}, a_{3},-a_{4}\right\} \\
\end{array}$ \\
\hline $\begin{array}{l}{[\text { CDM-F; Null }]} \\
\text { with equi-spaced pilots } \\
N=32, L_{0}=2, M_{0}=16 \\
K=1, N_{\mathrm{Tx}}=4\end{array}$ & $\begin{array}{l}0 \\
1 \\
2\end{array}$ & $\begin{array}{l}0 \\
0 \\
0 \\
0\end{array}$ & $\begin{array}{c}\mathcal{J}_{i}^{\text {pilot }}=\{1,5,9,13,17,21,25,29,\} \\
\mathcal{J}_{i}^{\text {null }}=\{3,7,11,15,19,23,27,31\} \\
c_{0}\left[k \in \mathcal{J}_{i}^{\text {pilot }}\right]=\left\{a_{1}, a_{2}, a_{3}, a_{4}, a_{5}, a_{6}, a_{7}, a_{8}\right\} \\
c_{1}\left[k \in \mathcal{J}_{i}^{\text {pilot }}\right]=\left\{a_{1} e^{j \pi / 16}, a_{2} e^{j 5 \pi / 16}, a_{3} e^{j 9 \pi / 16}, a_{4} e^{j 13 \pi / 16},\right. \\
\left.a_{4} e^{j-15 \pi / 16}, a_{3} e^{j-11 \pi / 16}, a_{2} e^{j-7 \pi / 16}, a_{1} e^{j-3 \pi / 16}\right\} \\
c_{2}\left[k \in \mathcal{J}_{i}^{\text {pilot }}\right]=\left\{a_{1} e^{j \pi / 8}, a_{2} e^{j 5 \pi / 8}, a_{3} e^{j-7 \pi / 8}, a_{4} e^{j-3 \pi / 8},\right. \\
\left.a_{4} e^{j \pi / 8}, a_{3} e^{j 5 \pi / 8}, a_{2} e^{j-7 \pi / 8}, a_{1} e^{j-3 \pi / 8}\right\} \\
c_{3}\left[k \in \mathcal{J}_{i}^{\text {pilot }}\right]=\left\{a_{1} e^{j 3 \pi / 8}, a_{2} e^{j-\pi / 8}, a_{3} e^{j-3 \pi / 8}, a_{4} e^{j 7 \pi / 8},\right. \\
\left.a_{4} e^{j 3 \pi / 8}, a_{3} e^{j-\pi / 8}, a_{2} e^{j-3 \pi / 8}, a_{1} e^{j 7 \pi / 8}\right\} \\
\end{array}$ \\
\hline $\begin{array}{c}{[\text { FDM } ; \text { Null }]} \\
N=16, L_{0}=2, M_{0}=8 \\
K=1, N_{\mathrm{Tx}}=2\end{array}$ & $\begin{array}{l}0 \\
1 \\
\end{array}$ & $\begin{array}{l}0 \\
0\end{array}$ & $\begin{array}{c}\mathcal{J}_{i}^{\text {pilot }}=\{1,3,9,11\} \\
\mathcal{J}_{i}^{\text {null }}=\{5,7,13,15\} \\
c_{0}\left[k \in \mathcal{J}_{i}^{\text {pilot }}\right]=\left\{a_{1}, 0, a_{2}, 0\right\} \\
c_{1}\left[k \in \mathcal{J}_{i}^{\text {pilot }}\right]=\left\{0, a_{3}, 0, a_{4}\right\}\end{array}$ \\
\hline $\begin{aligned} & {[\text { TFDM } ; \text { Null } / \mathrm{C}-\mathrm{F}] } \\
N= & 64, L_{0}=2, M_{1}=16 \\
& K=2, N_{\mathrm{Tx}}=3\end{aligned}$ & $\begin{array}{c}0 \\
0 \\
1 \\
--\frac{1}{2}-- \\
--\frac{2}{2}-- \\
\text { (alternative) } \\
\text { (alternative) } \\
\end{array}$ & $\begin{array}{c}0 \\
1 \\
0 \\
-\frac{1}{0}- \\
-\frac{0}{0}- \\
-\frac{1}{0}- \\
1\end{array}$ & 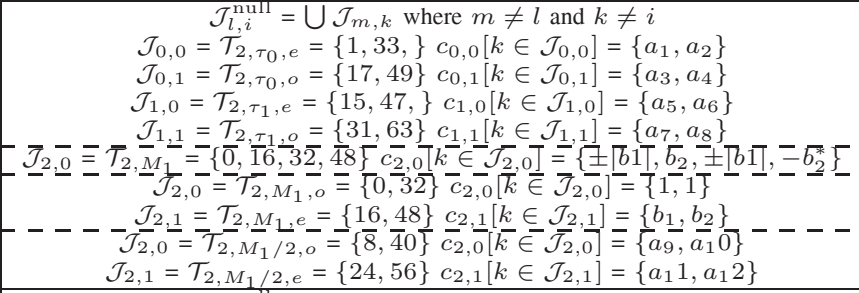 \\
\hline $\begin{array}{l}\text { [TFDM / CDM-T; Null / C-T] } \\
N=64, L_{0}=4, M_{0}=16 \\
\quad K=4, N_{\mathrm{Tx}}=5\end{array}$ & $\begin{array}{c}0 \\
0 \\
0 \\
0 \\
1 \\
1 \\
1 \\
1 \\
2 \\
2 \\
2 \\
2 \\
3 \\
3 \\
3 \\
--\frac{3}{4}-- \\
4 \\
-\frac{4}{4}-- \\
\text { (alternative) } \\
\end{array}$ & $\begin{array}{c}0 \\
1 \\
2 \\
3 \\
0 \\
1 \\
2 \\
3 \\
0 \\
1 \\
2 \\
3 \\
0 \\
1 \\
2 \\
3 \\
-- \\
- \\
- \\
1 \\
0 \\
0\end{array}$ & 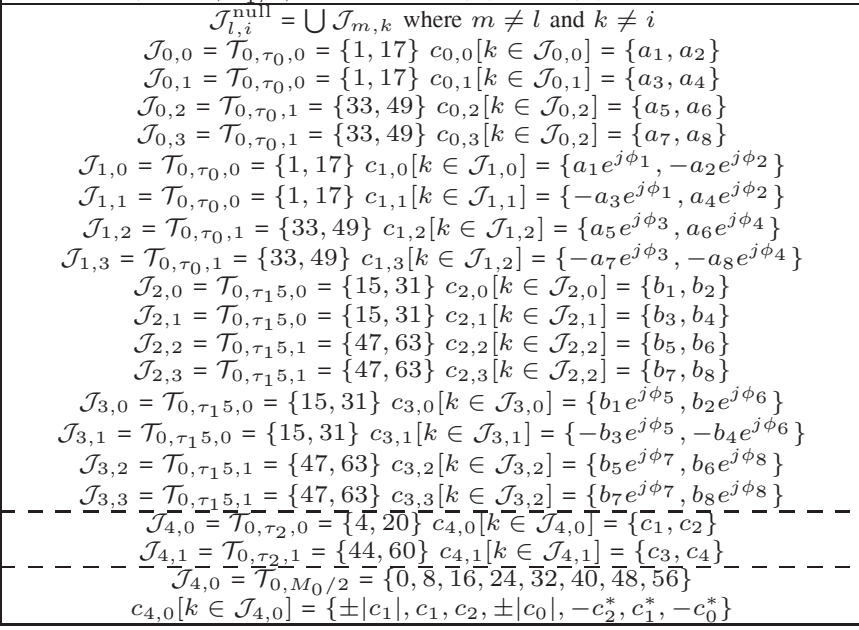 \\
\hline
\end{tabular}


TABLE II

EXAMPles of Proposed Pilot Designs ConT’D.

\begin{tabular}{|c|c|c|c|}
\hline Design & $\begin{array}{l}\text { antenna } \\
\text { index } i\end{array}$ & $\begin{array}{l}\text { symbol } \\
\text { index } l\end{array}$ & Pilots \\
\hline $\begin{array}{c}\text { [CDM-F; C-T] } \\
N=64, L_{0}=4, M_{0}=16 \\
K=2, N_{\mathrm{Tx}}=2\end{array}$ & $\begin{array}{l}0 \\
0 \\
1 \\
1\end{array}$ & $\begin{array}{l}0 \\
1 \\
0 \\
1\end{array}$ & $\begin{array}{c}\mathcal{J}_{i}=\{4,12,20,28,36,44,52,60\} \\
c_{0,0}\left[k \in \mathcal{J}_{i}\right]=\left\{a_{1}, a_{2}, a_{3}, a_{4}, a_{5}, a_{6}, a_{7}, a_{8}\right\} \\
c_{0,1}\left[k \in \mathcal{J}_{i}\right]=\left\{j a_{1}, j a_{2}, j a_{3}, j a_{4}, j a_{5}, j a_{6}, j a_{7}, j a_{8}\right\} \\
c_{1,0}\left[k \in \mathcal{J}_{i}\right]=\left\{a_{1},-a_{2}, a_{3},-a_{4}, a_{5},-a_{6}, a_{7},-a_{8}\right\} \\
c_{1,1}\left[k \in \mathcal{J}_{i}\right]=\left\{j a_{1},-j a_{2}, j a_{3},-j a_{4}, j a_{5},-j a_{6}, j a_{7},-j a_{8}\right\}\end{array}$ \\
\hline $\begin{array}{c}\text { [FDM; C-T] } \\
N=64, L_{0}=4, M_{0}=16 \\
K=2, N_{\mathrm{Tx}}=2\end{array}$ & $\begin{array}{l}0 \\
0 \\
1 \\
1\end{array}$ & $\begin{array}{l}0 \\
1 \\
0 \\
1\end{array}$ & $\begin{array}{c}\mathcal{J}_{i}=\{4,12,20,28,36,44,52,60\} \\
c_{0,0}\left[k \in \mathcal{J}_{i}\right]=\left\{a_{1}, 0, a_{2}, 0, a_{3}, 0, a_{4}, 0\right\} \\
c_{0,1}\left[k \in \mathcal{J}_{i}\right]=\left\{j a_{1}, 0, j a_{2}, 0, j a_{3}, 0, j a_{4}, 0\right\} \\
c_{1,0}\left[k \in \mathcal{J}_{i}\right]=\left\{0, a_{5}, 0, a_{6}, 0, a_{7}, 0, a_{8}\right\} \\
c_{1,1}\left[k \in \mathcal{J}_{i}\right]=\left\{0, j a_{5}, 0, j a_{6}, 0, j a_{7}, 0, j a_{8}\right\}\end{array}$ \\
\hline $\begin{array}{c}{[\text { FDM; C-T] }} \\
(\text { alternative }) \\
N=64, L_{0}=4, M_{0}=16 \\
K=2, N_{\mathrm{Tx}}=2\end{array}$ & $\begin{array}{l}0 \\
0 \\
1 \\
1\end{array}$ & $\begin{array}{l}0 \\
1 \\
0 \\
1\end{array}$ & $\begin{array}{c}\mathcal{J}_{i}=\{4,12,20,28,36,44,52,60\} \\
c_{0,0}\left[k \in \mathcal{J}_{i}\right]=\left\{a_{1}, 0, a_{2}, 0, a_{3}, 0, a_{4}, 0\right\} \\
c_{0,1}\left[k \in \mathcal{J}_{i}\right]=\left\{a_{1}, 0, a_{2}, 0,-a_{3}, 0,-a_{4}, 0\right\} \\
c_{1,0}\left[k \in \mathcal{J}_{i}\right]=\left\{0, a_{5}, 0, a_{6}, 0, a_{7}, 0, a_{8}\right\} \\
c_{1,1}\left[k \in \mathcal{J}_{i}\right]=\left\{0, a_{5}, 0, a_{6}, 0,-a_{7}, 0,-a_{8}\right\}\end{array}$ \\
\hline $\begin{array}{c}{[\text { FDM; C-T }]} \\
(\text { alternative }) \\
N=64, L_{0}=4, M_{0}=16 \\
K=2, N_{\mathrm{Tx}}=2\end{array}$ & $\begin{array}{l}0 \\
0 \\
1 \\
1\end{array}$ & $\begin{array}{l}0 \\
1 \\
0 \\
1\end{array}$ & $\begin{array}{c}\mathcal{J}_{i}=\{4,12,20,28,36,44,52,60\} \\
c_{0,0}\left[k \in \mathcal{J}_{i}\right]=\left\{a_{1}, 0, a_{2}, 0, a_{3}, 0, a_{4}, 0\right\} \\
c_{0,1}\left[k \in \mathcal{J}_{i}\right]=\left\{a_{1}, 0, a_{2}, 0, a_{3}, 0, a_{4}, 0\right\} \\
c_{1,0}\left[k \in \mathcal{J}_{i}\right]=\left\{0, a_{5}, 0, a_{6}, 0, a_{7}, 0, a_{8}\right\} \\
c_{1,1}\left[k \in \mathcal{J}_{i}\right]=\left\{0,-a_{5}, 0,-a_{6}, 0,-a_{7}, 0,-a_{8}\right\}\end{array}$ \\
\hline $\begin{array}{c}{[\text { TDM } ; \text { Null }]} \\
N=16, L_{0}=2, M_{0}=8 \\
K=2, N_{\mathrm{Tx}}=2\end{array}$ & $\begin{array}{l}0 \\
1\end{array}$ & $\begin{array}{l}0 \\
1\end{array}$ & $\begin{array}{c}\mathcal{J}_{i}^{\text {pilot }}=\{1,2,9,10\} \\
\mathcal{J}_{i}^{\text {null }}=\{6,7,14,15\} \\
c_{0,0}\left[k \in \mathcal{J}_{i}^{\text {pilot }}\right]=\left\{a_{1}, a_{2}, a_{3}, a_{4}\right\} \\
c_{1,1}\left[k \in \mathcal{J}_{i}^{\text {pilot }}\right]=\left\{a_{1}, a_{2}, a_{3}, a_{4}\right\}\end{array}$ \\
\hline
\end{tabular}

\section{C. [CDM-T; C-T] Design With Self-Mirror Tones}

The C-T design in the above subsection uses $\boldsymbol{v}_{l}$ and $\boldsymbol{v}_{m}$ with $l \neq m$ for tones $k$ and $-k$. This approach becomes irrelevant for self-mirror tones (i.e., for $\left.k=(-k)_{N}\right)$. When the pilots contain self-mirror tones, the index sets are given by

$$
\begin{aligned}
\mathcal{J}_{l, i} & =\left\{0, M_{0}, 2 M_{0}, \ldots, N-M_{0}\right\}, \forall i, l \\
\mathcal{I}_{l} & =\{0,1,2, \ldots, N-1\} \backslash \mathcal{J}_{l} .
\end{aligned}
$$

For non-self-mirror tones, the C-T design from the previous subsection is applied with $K$ defined below. At self-mirror tones $k=0$ and $N / 2$, we present two designs. The first design is given by

$$
\begin{aligned}
& \boldsymbol{c}_{0}[k]=e^{j \phi_{0, k}} \boldsymbol{v}_{n}^{\prime}, n \in\{1, \ldots, K-1\} \\
& \boldsymbol{c}_{i}[k]=e^{j \phi_{i, k}} \operatorname{diag}\left\{\boldsymbol{v}_{m_{i}}\right\} \boldsymbol{c}_{0}^{*}[k], i=1, \ldots, N_{\mathrm{Tx}}-1
\end{aligned}
$$

where $\left\{\phi_{i, k}\right\}$ are arbitrary, $\left[\boldsymbol{v}^{\prime}{ }_{n}\right]_{k} \triangleq \sqrt{\left[\boldsymbol{v}_{n}\right]_{k}}$ and the indexes $n$ and $m_{i}$ satisfy i) $\left(m_{i}+m_{j}-n\right)_{K} \neq 0, m_{i}, m_{j} \in$ $\left\{0,1, \ldots, N_{\text {Tx }}-1\right\}, m_{i} \neq m_{j}$ if $i \neq j$, ii) $\left(n-m_{i}\right)_{K} \neq 0$, iii) $\left(m_{i}-m_{j}\right)_{K} \neq 0, \forall m_{i} \neq m_{j}$. For simplicity, we can set $n=1$ in the above equations which yields $2 \leq m, l \leq K / 2$ and $K=2 N_{\text {Tx }} . K$ can also be $2\left(N_{\text {Tx }}+\tau\right)$ with $\tau \in\{0,1,2, \ldots\}$.

The second design at self-mirror tones is defined by

$$
\begin{aligned}
& \boldsymbol{c}_{0}[k]=e^{j \phi_{0, k}} \boldsymbol{v}_{n}, n \in\{1, \ldots, K-1\} \& n \neq K / 2 \\
& \boldsymbol{c}_{i}[k]=e^{j \phi_{i, k}} \operatorname{diag}\left\{\boldsymbol{v}_{m_{i}}\right\} \boldsymbol{c}_{0}^{*}[k], \quad i=1, \ldots, N_{\mathrm{Tx}}-1
\end{aligned}
$$

with the indexes $n$ and $m_{i}$ satisfying i) $m_{i} \neq n,\left(m_{i}-\right.$ $n)_{K / 2} \neq 0$, ii) $\left(m_{i}+m_{j}-2 n\right)_{K} \neq 0, m_{i}, m_{j} \in$ $\left\{0,1, \ldots, N_{\mathrm{Tx}}-1\right\}, m_{i} \neq m_{j}$ if $i \neq j$, iii) $\left(2 n-m_{i}\right)_{K} \neq 0$, and $\left\{\phi_{i, k}\right\}$ are arbitrary. By simply setting $n=1$, we have $3 \leq m \leq K / 2$ and $K=2\left(N_{\mathrm{Tx}}+\tau\right)$ with $\tau \in\{1,2, \ldots\}$. Using $M_{m}$ in place of $M_{0}$ will require more overhead.

\section{D. [CDM-F; Null] Design}

This design uses $V L_{m}$ constant amplitude pilot tones with the index set $\mathcal{J}_{0, i}^{\text {pilot }}=\mathcal{J}_{0}^{\text {pilot }}$ and $V L_{m}$ null tones with the index set $\mathcal{J}_{0, i}^{\text {null }}=\mathcal{J}_{0}^{\text {null }}$ where $\mathcal{J}_{0}^{\text {pilot }}$ and $\mathcal{J}_{0}^{\text {null }}$ are mirrors of each other, and $V \geq N_{\mathrm{Tx}}$ and $V L_{m} \leq N / 2$. Self-mirror tones cannot be used. Define $\mathcal{T}_{n, k} \triangleq\left[k, k+M_{n}, k+2 M_{n}\right.$, $\left.\ldots, k+N-M_{n}\right]$ which consists of cyclically equi-spaced $L_{n}$ indexes from $[0, N-1]$. Then the index sets are given by

$$
\begin{aligned}
\mathcal{J}_{0, i}^{\text {pilot }} & =\mathcal{J}_{0}^{\text {pilot }}=\bigcup_{k=0}^{V-1} \mathcal{T}_{n, \tau_{k}} \\
\mathcal{J}_{0, i}^{\text {null }} & =\mathcal{J}_{0}^{\text {null }}=\left\{N-\mathcal{J}_{0}^{\text {pilot }}\right\} \\
\mathcal{I}_{l} & =\{0,1, \ldots, N-1\} \backslash\left\{\mathcal{J}_{0}^{\text {pilot }} \cup \mathcal{J}_{0}^{\text {null }}\right\}
\end{aligned}
$$

where $\tau_{k} \in\left\{\left\{1,2, \ldots, M_{n}-1\right\} \backslash\left\{M_{n} / 2\right\}\right\}, \tau_{k} \neq \tau_{n}$ if $k \neq$ $n,\left\{\tau_{k}\right\} \cap\left\{M_{n}-\tau_{k}\right\}=\emptyset$, and $M_{n} \geq 2 N_{\mathrm{Tx}}+2$.

Due to the mirror null tones, the I/Q imbalance induced interferences are completely suppressed. The pilots of different antennas are decoupled by CDM-F design as

$$
\begin{aligned}
& \boldsymbol{c}_{0}[k]=e^{j \phi_{k}}, \text { arbitrary } \phi_{k}, k \in \mathcal{J}_{0}^{\text {pilot }} \\
& \boldsymbol{c}_{i}[k]=e^{\frac{j 2 \pi m i}{K}} \boldsymbol{c}_{0}[k], k \in \mathcal{T}_{n, \tau_{m}}, i \in\left\{1, \ldots, N_{\mathrm{Tx}}-1\right\} \\
& \boldsymbol{c}_{i}[k]=0, k \notin \mathcal{J}_{0}^{\text {pilot }}, i \in\left\{0, \ldots, N_{\mathrm{Tx}}-1\right\} .
\end{aligned}
$$

If the elements of $\mathcal{J}_{0}^{\text {pilot }}$ are cyclically equi-spaced, the pilots can also be given by

$$
\begin{aligned}
\boldsymbol{c}_{0}[k]= & e^{j \phi_{0, k}}, k \in \mathcal{J}_{0}^{\text {pilot }} \\
\boldsymbol{c}_{i}[k]= & e^{j \phi_{i}} e^{j 2 \pi k \tau_{i} / N} \boldsymbol{c}_{0}[k], i \in\left\{1,2, \ldots, N_{\mathrm{Tx}}-1\right\} \\
& L \leq \tau_{1} \leq L_{n}, L \leq \tau_{i+1}-\tau_{i} \leq L_{n} \\
\boldsymbol{c}_{i}[k]= & 0, k \notin \mathcal{J}_{0}^{\text {pilot }}, i \in\left\{0,1, \ldots, N_{\mathrm{Tx}}-1\right\}
\end{aligned}
$$

where $\left\{\phi_{i}, \phi_{0, k}\right\}$ are arbitrary phases. The choice of $V=N_{\mathrm{Tx}}$ and $L_{m}=L_{0}$ requires minimum overhead. Note that for $K=$ 1 , the maximum number of transmit antennas this design can support is $\frac{N}{2 L_{0}}-1$ since the null design cannot be applied to $\mathcal{T}_{1,0}$ which contains mirror pairs. However, the antenna $\frac{N}{2 L_{0}}$ can transmit pilots on $\mathcal{T}_{1,0}$ using C-F design. 


\section{E. [FDM; Null] Design}

This design decouples pilots of different antennas through FDM and suppresses mirror tone interferences by means of mirror null tones. It uses $K=1$ symbol with $N_{\mathrm{Tx}} L_{m} \leq N / 2$, and consists of $N_{\mathrm{Tx}} L_{m}$ constant amplitude pilot tones with the index set $\mathcal{J}_{0}^{\text {pilot }}=\cup_{i} \mathcal{J}_{0, i}^{\text {pilot }}$ and $N_{\text {Tx }} L_{m}$ null tones with the index set $\mathcal{J}_{0}^{\text {null }}$. The differences from [CDM-F; Null] are (i) the constant amplitude pilots can be arbitrary within and across antennas in this design while they are dependent across antennas in [CDM-F; Null] and (ii) a different antenna transmits its constant amplitude pilots only on distinct cyclically equi-spaced $L_{m}$ tones in this design while each antenna uses the same $N_{\mathrm{Tx}} L_{m}$ tones in [CDM-F; Null]. The index sets of [FDM; Null] design are defined by

$$
\begin{aligned}
\mathcal{J}_{0, i}^{\text {pilot }} & =\mathcal{T}_{n, \tau_{i}}, \tau_{k} \neq \tau_{n} \text { if } k \neq n \\
\mathcal{J}_{0, i}^{\text {null }} & =\mathcal{J}_{0}^{\text {null }}=\left\{N-\mathcal{J}_{0}^{\text {pilot }}\right\} \\
\mathcal{I}_{l} & =\{0,1, \ldots, N-1\} \backslash\left\{\mathcal{J}_{0}^{\text {pilot }} \cup \mathcal{J}_{0}^{\text {null }}\right\}
\end{aligned}
$$

where $\tau_{k} \in\left\{\left\{1,2, \ldots, M_{n}-1\right\} \backslash\left\{M_{n} / 2\right\}\right\},\left\{\tau_{k}\right\} \cap\left\{M_{n}-\right.$ $\left.\tau_{k}\right\}=\emptyset$. The pilot tones are given by

$$
\begin{aligned}
& \boldsymbol{c}_{i}[k]=e^{j \phi_{i, k}}, k \in \mathcal{J}_{0, i}^{\text {pilot }}, i \in\left\{0, \ldots, N_{\mathrm{Tx}}-1\right\} \\
& \boldsymbol{c}_{i}[k]=0, k \notin \mathcal{J}_{0, i}^{\text {pilot }}
\end{aligned}
$$

where $\left\{\phi_{i, k}\right\}$ are arbitrary. For $K=1$, the maximum number of transmit antennas this design can support is $\frac{N}{2 L_{0}}-1$ which is the same scenario as discussed in [CDM-F; Null] design.

\section{F. [TFDM; Null / C-F] Design}

In this design, pilots of different antennas are decoupled by TFDM design, while intra-antenna mirror tone interferences are addressed by mirror null tones, and inter-antenna mirror tone interferences are suppressed by code design across frequency domain (C-F) or mirror null tones across different antennas. This design uses $K=2$ symbols over which non-zero pilots (with the index set $\mathcal{T}_{n, \tau_{i}}$ ) of each antenna $i$ are spread out evenly. It requires $N_{\mathrm{Tx}} L_{n} \leq N$, and $L_{n} \geq 2 L_{0}$. The pilot index sets over two symbols for $(2 i)$ th and $(2 i+1)$ th antennas are chosen as $\mathcal{T}_{n, \tau_{2 i}}$ and $\mathcal{T}_{n, \tau_{2 i+1}}$ where $\tau_{k} \in\left\{\left\{1,2, \ldots, M_{n}-1\right\} \backslash\left\{M_{n} / 2\right\}\right.$ and $\tau_{2 i}+\tau_{2 i+1}=M_{n}$. If $N_{\mathrm{Tx}}$ is an odd number, the pilot index set over the two symbols for the last antenna $\left(i=N_{\mathrm{Tx}}-1\right)$ is $\mathcal{T}_{n, M_{n} / 2}$ which consists of mirror pairs excluding self-mirror tones. $\mathcal{T}_{n, \tau_{k}}$ is divided into two decimated sets $\mathcal{T}_{n, \tau_{k}, e}$ and $\mathcal{T}_{n, \tau_{k}, o}$, consisting of even elements and odd elements (their values can be even or odd) of $\mathcal{T}_{n, \tau_{k}}$, respectively. For each antenna pairs $2 i$ and $2 i+1, \mathcal{T}_{n, \tau_{2 i}, e}$ and $\mathcal{T}_{n, \tau_{2 i+1}, o}$ form mirror pairs, and so do $\mathcal{T}_{n, \tau_{2 i}, o}$ and $\mathcal{T}_{n, \tau_{2 i+1}, e}$. Antennas $2 i$ and $2 i+1$ transmit pilots on $\mathcal{T}_{n, \tau_{2 i}, e}$ and $\mathcal{T}_{n, \tau_{2 i+1}, o}$, respectively, in the first symbol, and $\mathcal{T}_{n, \tau_{2 i}, o}$ and $\mathcal{T}_{n, \tau_{2 i+1}, e}$, respectively, in the second symbol. Mathematically, the index sets are given by

$$
\begin{aligned}
& \mathcal{J}_{0,2 i}^{\text {pilot }}=\mathcal{T}_{n, \tau_{2 i}, e}, \quad \mathcal{J}_{0,2 i+1}^{\text {pilot }}=\mathcal{T}_{n, \tau_{2 i+1}, o} \\
& \mathcal{J}_{1,2 i}^{\text {pilot }}=\mathcal{T}_{n, \tau_{2 i}, o}, \quad \mathcal{J}_{1,2 i+1}^{\text {pilot }}=\mathcal{T}_{n, \tau_{2 i+1}, e} \\
& \mathcal{T}_{n, \tau_{2 i+1}, o}=\left\{N-\mathcal{T}_{n, \tau_{2 i}, e}\right\} \\
& \mathcal{T}_{n, \tau_{2 i+1}, e}=\left\{N-\mathcal{T}_{n, \tau_{2 i}, o}\right\}
\end{aligned}
$$

$$
\mathcal{J}_{0, N_{\mathrm{Tx}}-1}^{\text {pilot }}=\mathcal{T}_{n, M_{n} / 2, e} \& \mathcal{J}_{1, N_{\mathrm{Tx}}-1}^{\text {pilot }}=\mathcal{T}_{n, M_{n} / 2, o} \text {, odd } N_{\mathrm{Tx}}
$$

$$
\begin{aligned}
& \mathcal{T}_{n, N_{\mathrm{Tx}}-1, e}=\left\{N-\mathcal{T}_{n, N_{\mathrm{Tx}}-1, o}\right\}, \text { odd } N_{\mathrm{Tx}} \\
& \mathcal{I}_{l}=\{0, \ldots, N-1\} \backslash \mathcal{J}_{l}^{\text {pilot }}, \text { even } N_{\mathrm{Tx}} \\
& \mathcal{I}_{l}=\{0, \ldots, N-1\} \backslash\left\{\mathcal{J}_{l}^{\text {pilot }} \cup \mathcal{T}_{n, N_{\mathrm{Tx}}-1}\right\}, \text { odd } N_{\mathrm{Tx}} .
\end{aligned}
$$

For each antenna pair $(2 i, 2 i+1)$, the C-F design is given by

$$
\begin{gathered}
c_{2 i, 0}\left[\left[\mathcal{T}_{n, \tau_{2 i}, e}\right]_{m}\right]=e^{j \phi_{0, m}}, m=0,1, \ldots, L_{n-1}-1 \\
c_{2 i+1,0}\left[\left[\mathcal{T}_{n, \tau_{2 i+1}, o}\right]_{L_{n-1}-m}\right]=e^{j 2 \pi \lambda 2 m / L_{n}} e^{-j \phi_{0, m}} \\
\quad \lambda \in\left\{L, L+1, \ldots, L_{n-1}-L\right\} \\
c_{2 i, 1}\left[\left[\mathcal{T}_{n, \tau_{2 i}, o}\right]_{m}\right]=e^{j \phi_{1, m}}, m=0,1, \ldots, L_{n-1}-1 \\
c_{2 i+1,1}\left[\left[\mathcal{T}_{n, \tau_{2 i+1}, e}\right]_{L_{n-1}-m}\right]=e^{j 2 \pi \alpha(2 m+1) / L_{n}} e^{-j \phi_{1, m}}, \\
\alpha \in\left\{L, L+1, \ldots, L_{n-1}-L\right\}
\end{gathered}
$$

where $\left\{\phi_{0, m}\right\}$ and $\left\{\phi_{1, m}\right\}$ are arbitrary. For different antenna pairs, $\left\{\phi_{0, m}\right\},\left\{\phi_{1, m}\right\}, \lambda$, and $\alpha$ can be independently chosen. For an odd $N_{\mathrm{Tx}}, c_{N_{\mathrm{Tx}}-1,0}\left[k \in \mathcal{T}_{n, M_{n} / 2, o}\right]$ and $c_{N_{\mathrm{Tx}}-1,1}[k \in$ $\left.\mathcal{T}_{n, M_{n} / 2, e}\right]$ can be set to arbitrary unit amplitude symbols. At each of the two symbols, this design uses $N_{\mathrm{Tx}} L_{n-1}$ pilot tones for an even $N_{\mathrm{Tx}}$ and $\left(N_{\mathrm{Tx}}-1\right) L_{n-1}+L_{n}$ pilot tones (including null tones) for an odd $N_{\mathrm{Tx}}$.

\section{G. [CDM-F or FDM; C-T] Design}

This design uses two OFDM symbols. In CDM-F, each antenna transmits $N_{\mathrm{Tx}} L_{n}$ constant-amplitude pilot tones in each symbol, while in FDM each antenna transmits $L_{n}$ constantamplitude pilot tones and $\left(N_{\mathrm{Tx}}-1\right) L_{n}$ null pilot tones in each symbol. These pilot indexes are all mirror-pairs (may include self-mirror tones). The index sets are given by

$$
\begin{aligned}
\mathcal{J}_{l} & =\bigcup_{m=0}^{N_{\mathrm{Tx}}-1} \mathcal{T}_{n, \tau_{m}}, \tau_{m} \in\left\{0,1, \ldots, M_{n}-1\right\}, \\
\mathcal{I}_{l} & =\{0,1,2, \ldots, N-1\} \backslash \mathcal{J}_{l}, \\
\mathcal{J}_{l, i} & = \begin{cases}\mathcal{J}_{l}, & \text { CDM-F } \\
\mathcal{J}_{l, i}=\mathcal{T}_{n, \tau_{i}}, & \text { FDM }\end{cases}
\end{aligned}
$$

where $\tau_{m} \neq \tau_{k}$ if $m \neq k$ and $\left\{\tau_{m}\right\}=\left\{\left(M_{n}-\tau_{m}\right)_{M_{n}}\right\}$.

The choice of $n=0$ in $L_{n}$ and $M_{n}$ yields minimum pilot overhead. The pilots from different antennas are decoupled by CDM-F or FDM, while the mirror tone interferences are suppressed by C-T over two symbols. For CDM-F, each antenna transmits constant amplitude pilots on $\mathcal{J}_{l}$. For FDM, the $m$ th antenna transmits constant amplitude pilots on $\mathcal{T}_{n, \tau_{m}}$ and null tones on $\left\{\mathcal{J}_{l} \backslash \mathcal{T}_{n, \tau_{m}}\right\}$. The C-T design is described by the relationship of the pilot vectors at the second symbol to those at the first symbol. For CDM-F, the pilot vectors at the second symbol are just $\sqrt{-1}$ times the corresponding pilot vectors at the first symbol. For FDM, we can have several approaches such as: (i) the pilot vectors at the second symbol are $\sqrt{-1}$ times those at the first symbol, (ii) the pilot tones with indexes less than $N / 2$ remain the same over two symbols, while the remaining pilots change polarities across the two symbols, or (iii) for each antenna pair with mirror index sets $\mathcal{T}_{n, \tau_{m}}$ and $\mathcal{T}_{n, \tau_{\bar{m}}}$, the antenna using $\mathcal{T}_{n, \tau_{m}}$ transmits the same pilots on both symbols, while the other antenna changes polarities of pilots across the two symbols. 


\section{H. [TFDM / CDM-T; Null / C-T] Design}

In this design, pilots from different antennas are decoupled through TFDM and CDM-T, while mirror tone interferences within each antenna are addressed by mirror null tones, and those across antennas are suppressed by mirror null tones and C-T. The antennas are divided into $K_{g}$ groups. Pilot tones of different groups are disjoint via TFDM (or TDM). Each antenna group $m$ (for an even $N_{\mathrm{Tx}}$ ) consists of an even number of antennas, say $2 N_{m}$, and uses $\mathcal{T}_{n, \tau_{m}}$ and $\mathcal{T}_{n, \tau_{\bar{m}}}$ where $\tau_{m} \in\left\{\left\{1,2, \ldots, M_{n}-1\right\} \backslash\left\{M_{n} / 2\right\}\right\}$, $\tau_{m} \neq \tau_{k}$ if $m \neq k, \bar{m}=M_{n}-m, \tau_{m}+\tau_{\bar{m}}=M_{n}$, and $n \in\left\{1, \ldots, \log _{2}\left(N /\left(2 K_{g} L_{0}\right)\right\} . \mathcal{T}_{n, \tau_{m}}\right.$ and $\mathcal{T}_{n, \tau_{\bar{m}}}$ are each divided into two subsets of the same cardinality, denoted by $\mathcal{T}_{n, \tau_{m}, 0}, \mathcal{T}_{n, \tau_{m}, 1}, \mathcal{T}_{n, \tau_{\bar{m}}, 0}$, and $\mathcal{T}_{n, \tau_{\bar{m}}, 1}$, such that $\mathcal{T}_{n, \tau_{m}, 0}$ and $\mathcal{T}_{n, \tau_{\bar{m}}, 1}$ form mirror pairs and so do $\mathcal{T}_{n, \tau_{m}, 1}$ and $\mathcal{T}_{n, \tau_{\bar{m}}, 0}$. This division need not be a splitting of even and odd elements as required in Section IV-F.

The first half of the antennas within group $m$ transmit on $\mathcal{T}_{n, \tau_{m}, 0}$ during the first $Q_{m}\left(\geq N_{m}\right)$ symbols and on $\mathcal{T}_{n, \tau_{m}, 1}$ during the next $Q_{m}$ symbols. The other half of the antennas transmit on $\mathcal{T}_{n, \tau_{\bar{m}}, 0}$ during the first $Q_{m}$ symbols and on $\mathcal{T}_{n, \tau_{\bar{m}}, 1}$ during the next $Q_{m}$ symbols. These two subsets of antennas within each group are of TFDM type. The pilot for antenna $i$ of group $m$ on corresponding subcarrier $\left[\mathcal{T}_{n, *, *}\right]_{k}$ at $l$ th symbol is given by $e^{j \phi_{i, k}} e^{j 2 \pi l i / Q_{m}} a_{m, l, k}$ where $\left\{\phi_{i, k}\right\}$ are arbitrary and $\left\{a_{m, l, k}\right\}$ are arbitrary constant amplitude symbols, i.e., antennas transmitting on the same subcarrier follow CDM-T design. Each group $m$ requires $2 L_{n}$ tones over $2 N_{m}$ symbols, and setting $n=0$ in $L_{n}$ and $M_{n}$ yields a smaller overhead.

For an odd $N_{\mathrm{Tx}}$, a dummy antenna can be fictitiously added in the design to have an even $N_{\mathrm{Tx}}$. Two more-efficient alternatives are described below where pilots for the first $N_{\mathrm{Tx}}-1$ (even) antennas are developed according to the abovementioned design. In the first alternative, the last antenna transmits pilots on $\mathcal{T}_{n, 0}$ with $n \geq 1$ over one symbol using C$\mathrm{F}$ design. In the second alternative, the last antenna transmits arbitrary constant amplitude pilots on $\mathcal{T}_{n, M_{n+1}}$ with $n \geq 0$ over two symbols using C-T design, or on $\mathcal{T}_{n, M_{n+1}, e}$ with $n \geq 0$ at the first symbol and on $\mathcal{T}_{n, M_{n+1}, o}$ at the second symbol (i.e., Null design over two symbols).

\section{I. [TDM; Null] Design}

In this design, pilots of different antennas are decoupled by means of TDM while mirror tone interferences are suppressed by means of null tones. All antennas transmit on the same set of subcarriers, but each antenna transmits during a different OFDM symbol. Thus, $K=N_{\text {Tx }}$ OFDM symbols are required, while data can be transmitted on other subcarriers in a pilot-data-multiplexed format. This design can handle a larger number of null guard tones than other designs. Let $\mathcal{J}^{\text {guard }}$ denote the tone index set for the null guard tones. First, obtain $\mathcal{T}_{n, m}$ such that $\left\{\mathcal{T}_{n, m} \cap \mathcal{J}^{\text {guard }}\right\}=\emptyset$, $m \in\left\{\left\{1,2, \ldots, M_{n}-1\right\} \backslash\left\{M_{n} / 2\right\}\right\}$. With $\bar{m} \triangleq M_{n}-m$, we have $\mathcal{T}_{n, \bar{m}}=\left\{N-\mathcal{T}_{n, m}\right.$ modulo $\left.N\right\}$. Then, the index sets are given by

$$
\begin{aligned}
& \mathcal{J}_{i, l}^{\text {pilot }}= \begin{cases}\mathcal{T}_{n, m}, & \text { if } i=l \\
\emptyset, & \text { else }\end{cases} \\
& \mathcal{J}_{i, l}^{\text {null }}= \begin{cases}\mathcal{T}_{n, \bar{m}}, & \text { if } i=l \\
\mathcal{T}_{n, m} \cup \mathcal{T}_{n, \bar{m}}, & \text { else }\end{cases} \\
& \mathcal{I}_{l}=\{0,1,2, \ldots, N-1\} \backslash\left\{\mathcal{T}_{n, m} \cup \mathcal{T}_{n, \bar{m}} \cup \mathcal{J}^{\text {guard }}\right\} .
\end{aligned}
$$

The non-zero pilot tones can have arbitrary phases $\left\{\phi_{i, k}\right\}$ as

$$
\boldsymbol{c}_{i, i}[k]=e^{j \phi_{i, k}}, k \in \mathcal{J}_{i, i}^{\text {pilot }}, i \in\left\{0,1, \ldots, N_{\mathrm{Tx}}-1\right\} .
$$

For minimum overhead and operability with largest number of null guard tones, $M_{n}$ should be set to $M_{0}$.

\section{J. Other Designs}

Other variations or combinations of the above designs are also possible. For example, [CDM-F; C-T] and [FDM; C$\mathrm{T}]$ designs can be combined as [CDM-F/FDM; C-T] where antennas are divided into two groups such that there is no mirror pair across the two groups, and the first group applies [CDM-F; C-T] while the other group uses [FDM; C-T]. Other combinations may also be possible, but for practical design simplicity we skip further investigation in this direction.

\section{Simulation Results and Discussions}

\section{A. Parameter Setting}

System parameters in the simulation are $N_{\mathrm{Tx}}=2, N_{\mathrm{Rx}}=$ $2, N=64,6$ left and 6 right null guard tones, $M$-ary QAM with $M=16$, and a Rayleigh fading channel having an exponential power delay profile ( $3 \mathrm{~dB}$ per tap decay factor) with 4 sample-spaced taps. The FI I/Q imbalances are set to $\alpha=\frac{a_{t}^{I}}{a_{t}^{Q}}=\frac{a_{r}^{I}}{a_{r}^{Q}}=1.09648(=0.4 \mathrm{~dB})$, and $\Delta \theta=\theta_{t}^{I}-\theta_{t}^{Q}$ $=\theta_{r}^{I}-\theta_{r}^{Q}=3^{\circ}{ }^{2}$. The FD I/Q imbalances are modeled by 3-tap filters (hence, $L=8$ ) with discrete-time impulse responses of $[0.01,0.9999,0.01]$ and $[0.015,0.9998,0.01]$ for the transmit I and Q branches, and [0.012, 0.9997, 0.018], and $[0.01,0.9997,0.02]$ for the receive I and Q branches. For performance comparison, we use the pilot design from [21] as SISO Reference. We use the design from [24] as MIMO Reference 1 and an FDM design from [11] ${ }^{3}$ as MIMO Reference 2. In all methods, the estimators from Eqns. (16) and (17) are utilized and for BER results the maximum likelihood (ML) detection (joint detection of mirror tones) is applied. The energies of a non-zero pilot tone in the SISO references and the MIMO reference 1 are set to be the same as the average bit energy of data. The total pilot energy is kept the same for all pilot designs in each case (SISO or MIMO). For the SISO results, the system parameters are the same except for the number of antennas.

\footnotetext{
${ }^{2}$ These values are within typical ranges (e.g., see [4], [22], [27], [28]).

${ }^{3}$ To illustrate the performance degradation when I/Q imbalance is not considered in the pilot design, we pick a particular FDM design. Some of the pilot designs from [11] with $2 N_{\mathrm{Tx}} L_{0}$ pilot tones may yield the same estimation performance as the proposed ones.
} 


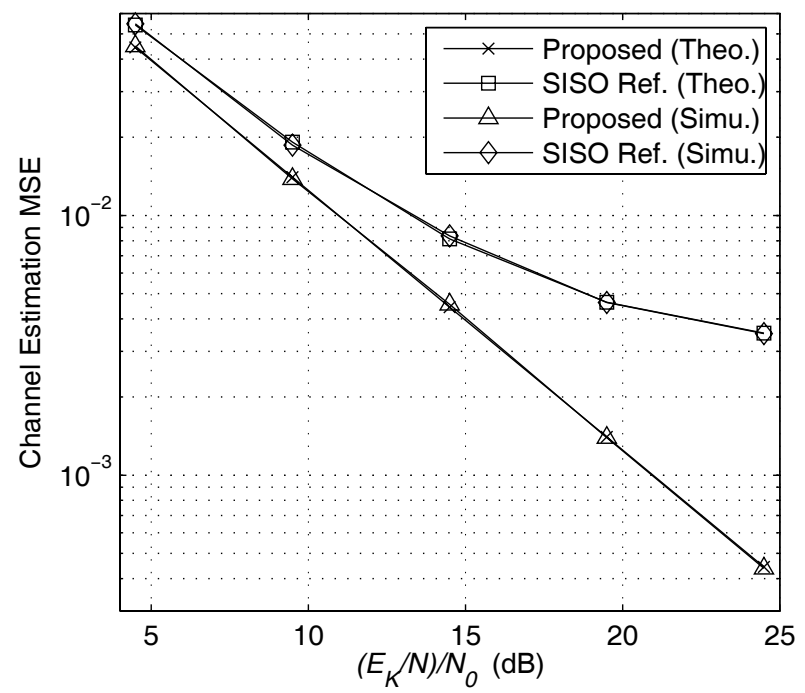

Fig. 3. Channel estimation MSE comparison of different pilot designs in a SISO OFDM system.

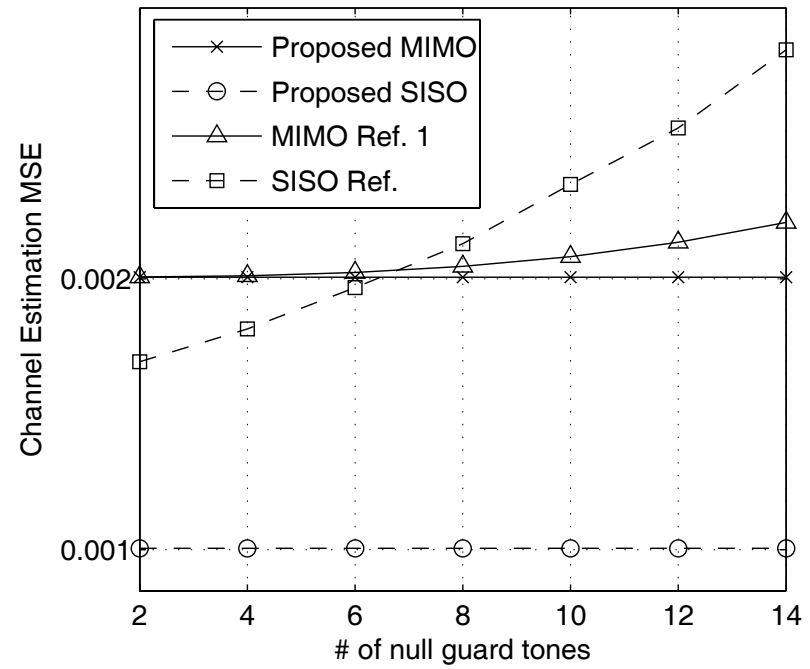

Fig. 4. Channel estimation MSE comparison of different pilot designs while varying the number of guard tones, $\left(\left(E_{K} / N\right) / N_{0}=21 \mathrm{~dB}\right)$

\section{B. Estimation and BER Performance}

Fig. 3 shows the channel estimation MSEs $\left(\mathrm{MSE}_{p}+\mathrm{MSE}_{q}\right)$ from simulation and the theoretical MSEs ((20) for the reference design and (30) for the proposed design) for a SISO system. The proposed design outperforms the SISO reference which experiences a flooring effect at high SNR. The nulling of some of the subcarriers results in a breakdown of the coding used in [21] to eliminate the mirror tone interferences and degrades the channel estimation MSE. The theoretical MSEs match the simulation MSEs very well.

The effects of guard tones on the pilot designs are illustrated in Fig. 4 using the theoretical MSEs. The MSE degradation of the SISO reference pilot design is observed to be more sensitive to the number of null guard tones than the MIMO Reference 1 which is due to different coding strategies adopted in the pilot designs. The larger MSE level of MIMO pilot

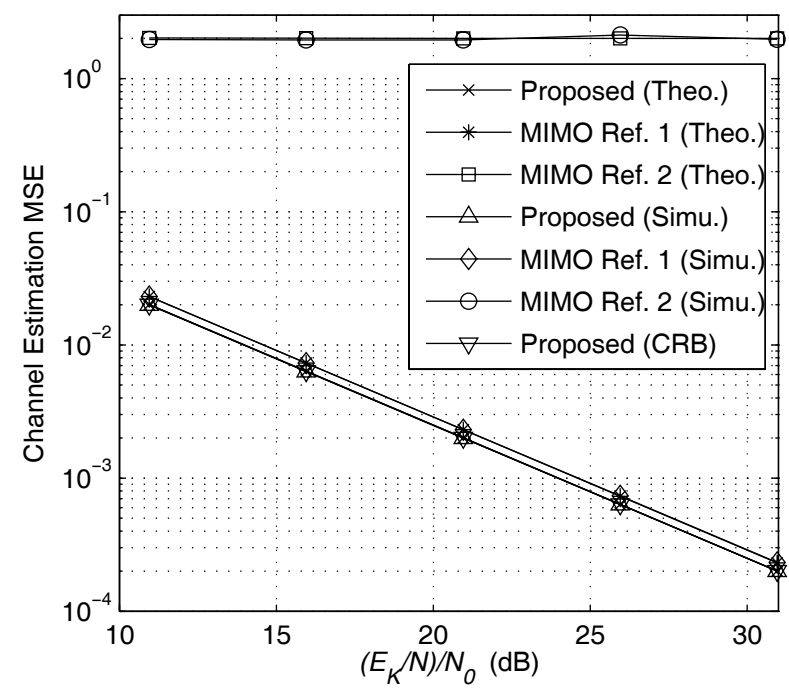

Fig. 5. Channel estimation MSE comparison of different pilot designs in a $2 \times 2$ MIMO OFDM system.

TABLE III

Pilot OVERHEAD COMPARISON

\begin{tabular}{||l|c||}
\hline Design & $\begin{array}{c}\text { Overhead } \\
\text { (\# of Tones) }\end{array}$ \\
\hline (MIMO) [TDM; TD/C-F] & $2 N_{\mathrm{Tx}} L_{0}$ \\
(MIMO) [CDM-T; C-T] & $2 N_{\mathrm{Tx}} L_{0}$ \\
(MIMO) [CDMF; Null] & $2 N_{\mathrm{Tx}} L_{0}$ \\
(MIMO) [FDM; Null] & $2 N_{\mathrm{Tx}} L_{0}$ \\
(MIMO) [TFDM; Null/C-F] & $4 L_{0}\left\lceil N_{\mathrm{Tx}} / 2\right\rceil$ \\
(MIMO) [TFDM/CDM-T; Null/C-T] & $2 N_{\mathrm{Tx}} L_{0}$ \\
(MIMO) [TDM; Null] & $2 N_{\mathrm{Tx}} L_{0}$ \\
(MIMO) Design from [24] & $2 N_{\mathrm{Tx}} N$ \\
\hline (SISO) Design from [21] & $N$ \\
\hline
\end{tabular}

designs if compared to the SISO designs is due to the fact that the MSE presented is the sum of MSEs of all parameters under estimation and that MSE increases with the number of parameters under estimation.

Fig. 5 presents the channel estimation MSEs from simulation, the theoretical MSEs, and the CRB from (31) for the MIMO system. The proposed design outperforms both reference designs. Although MIMO Reference 2 gives optimal estimation performance for systems without I/Q imbalances, it causes a substantial performance degradation in the presence of I/Q impairments. MIMO Reference 1 has a slight performance loss due to its non-optimality for systems with guard bands. The theoretical MSEs in Section III give an exact match to the simulation MSE results which show no noticeable difference from the CRB. This is due to the fact that the mild frequency-selectivity of the I/Q imbalance makes the white noise assumption used in the pilot design valid, and then the estimators become ML with the Gaussian signal model which are known to approach the CRB.

In Fig. 6, the (uncoded) BER performances of different pilot designs are compared for the MIMO system. Similar conclusions for the MSE performance apply for the BER performance. Although the BER improvement is small, the proposed pilot designs reduce the pilot overhead greatly (see 


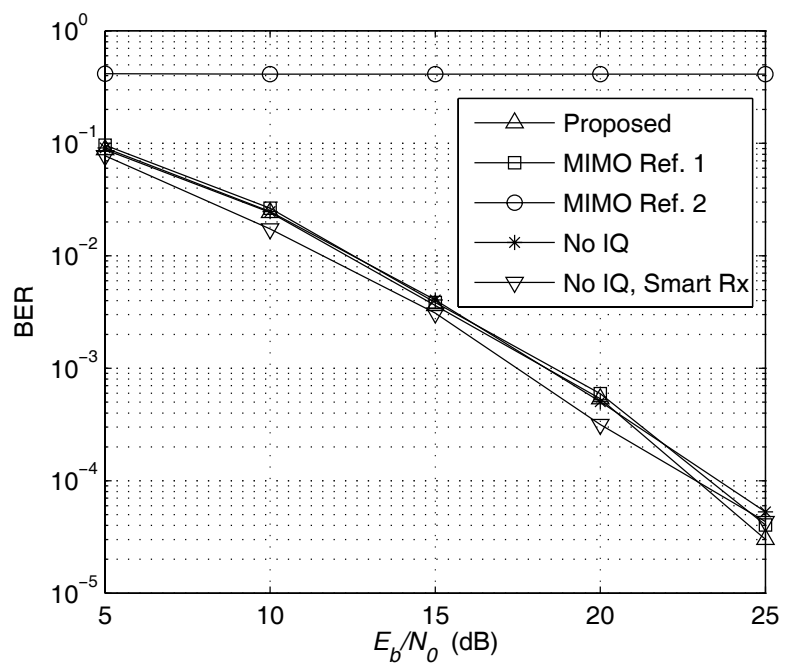

Fig. 6. BER comparison of different pilot designs for 16-QAM in a $2 \times 2$ MIMO OFDM system.

Table III) which increases the data capacity/throughput, and can also reduce the data detection latency if compared to MIMO Reference 1. When the receiver does not know the absence of I/Q imbalance, its performance is the same as the cases with $\mathrm{I} / \mathrm{Q}$ imbalance. But when the receiver knows the absence of I/Q imbalance (denoted as the Smart Rx), its performance is better than those with I/Q imbalance except at high SNR where the ML receiver gains frequency diversity provided by the I/Q imbalance.

Fig. 7 presents the MSE performance of the proposed pilot design under various FI I/Q imbalance levels. The low, medium, and high I/Q imbalance cases, respectively, correspond to $\left(\alpha=0.1 \mathrm{~dB}, \Delta \theta=1^{\circ}\right),\left(\alpha=0.7 \mathrm{~dB}, \Delta \theta=10^{\circ}\right)$, and $\left(\alpha=2 \mathrm{~dB}, \Delta \theta=30^{\circ}\right)^{4}$. The no I/Q imbalance case refers to $\left(\alpha=0 \mathrm{~dB}, \Delta \theta=0^{\circ}\right)$ and without FD I/Q imbalance, while the receiver still considers there is I/Q imbalance and estimates in the same way as in the other cases. For the Smart Rx case, the MSE drops proportional to the number of taps estimated. From Fig. 7, it is clear that the channel estimation MSE is not affected by the FI I/Q imbalance level.

In Fig. 8, to show the effect of different levels of FD I/Q imbalance, we have evaluated an additional set of FD I/Q imbalance filters with impulse responses of [0.9999, 0.01] and $[0.9999,0.015]$ for the transmit I and Q branches, and $[0.9998,0.018]$, and $[0.9999,0.01]$ for the receive I and Q branches. This corresponds to an equivalent channel with $L=6$ taps. We have also included the performance of the Smart Rx where the number of parameters under estimation is $L=4$ as opposed to $2 L=12$ and $2 L=16$ in the other two cases. The MSE gaps are due to the differences in the numbers of parameters under estimation. Hence, the frequency-selectivity level of I/Q imbalance (more specifically, the lengths of the equivalent direct and mirror channels) can affect the total channel estimation MSE.

\footnotetext{
${ }^{4}$ The values for the high case are outside of the current expected ranges for hardware in use today (see [27], [28]). However, as semiconductor downscaling continues, higher values for I/Q imbalance such as these could be seen.
}

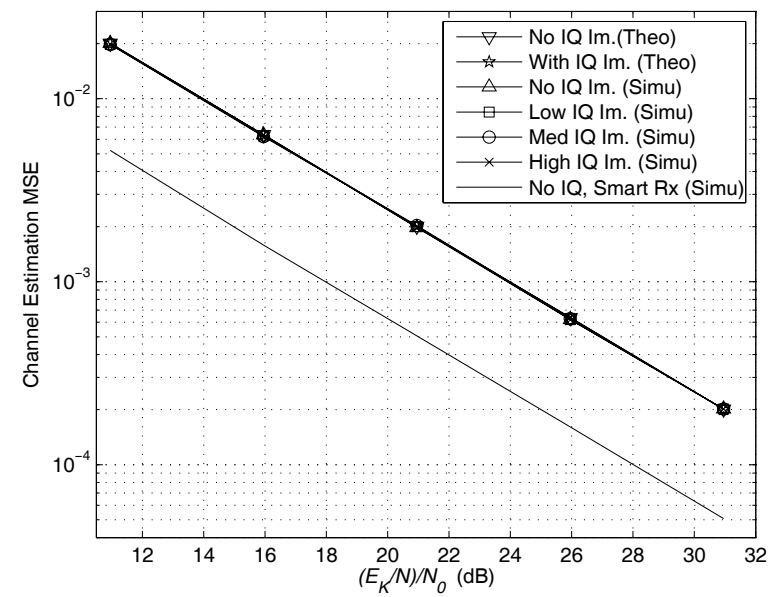

Fig. 7. Effects of different levels of FI I/Q imbalance on a proposed pilot design in a $2 \times 2$ MIMO OFDM system.

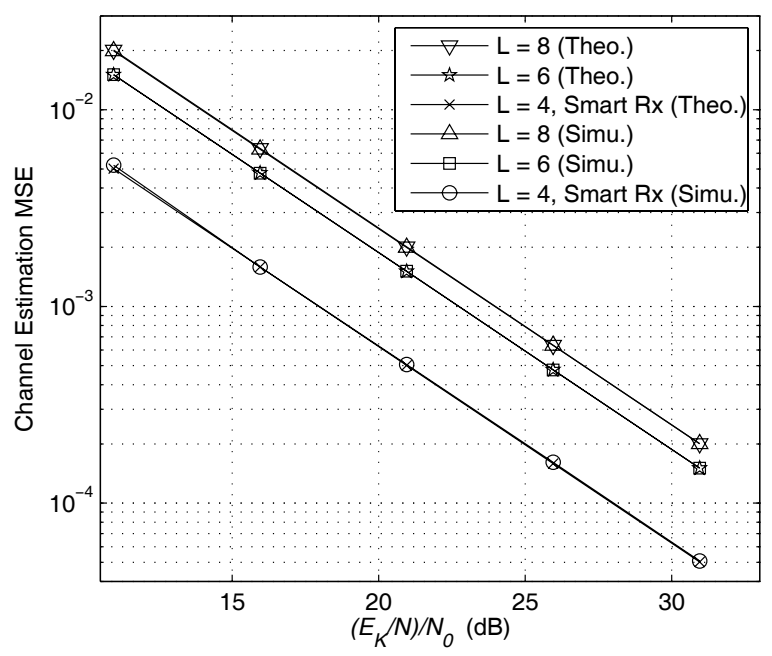

Fig. 8. Effects of different levels of FD I/Q imbalance on a proposed pilot design in a $2 \times 2$ MIMO OFDM system.

\section{Comparison in Other Aspects}

All of the proposed designs give the same estimation performance since they meet the criteria defined by (24). However, if other system constraints or impairments are considered, there could be advantages of one over another. For example, some designs such as [CDM-T; C-T] and [TDM; Null] are better equipped to facilitate a larger null guard band than others since the gaps between used subcarriers is larger. However, they utilize more OFDM symbols, and thus would introduce more latency at the receiver.

The proposed designs can be applied to preamble as well as pilot-data-multiplexed symbols because the design problem was formulated so. This reduces the pilot overhead and the latency at the receiver. The existing designs in [15], [16], [18]-[20], [22], [24] only apply for preamble since no data tones are considered in the designs. The proposed designs are applicable for both SISO and MIMO OFDM systems while the existing methods such as [15]-[22] only address for SISO OFDM systems. In term of the estimation performance, 
the proposed designs yield white-noise optimality and close to ideal-optimal results for colored noise at the demodulator output. The existing methods such as [19], [21], [22] only provide a suboptimal estimation performance which degrades for systems with guard bands while the proposed designs can overcome this shortfall as well. The comparison of pilot overhead for several pilot designs is presented in Table III. Under the considered system setting, the proposed designs require $1 / 4$ of the overhead of the SISO reference design, and $1 / 8$ of that of the MIMO Reference 1 .

\section{CONCLUSIONS}

We have developed efficient pilot designs for estimation of the equivalent channel responses incorporating FI and FD transmitter and receiver I/Q imbalances in MIMO OFDM systems. The receive filter output noise samples in the presence of FD receiver I/Q imbalance are colored and generally unknown, and hence development of exactly-optimal pilot designs is impractical. However, in practice the frequencyselectivity of I/Q imbalance is very small and hence our pilot designs developed based on the white noise condition are observed to yield essentially the same performance as the CRB. To be applicable in the pilot-data-multiplexed format, not only the data and pilot tones need to be disjoint but also each type (pilot or data) should occupy only on mirror subcarrier pairs. The minimum number of pilot tones required for the considered estimators is at least doubled if compared to the systems without I/Q imbalance. To suppress inter-antenna interferences, the pilots of different transmit antennas need to satisfy the condition in (42) as required in systems without I/Q imbalance but also an additional condition due to the mirror tone interference as given in (47).

We have also observed that the frequency-selectivity level of I/Q imbalance can affect the total channel estimation MSE while the FI I/Q imbalance level does not. The proposed pilot designs are more efficient than the existing designs in terms of overhead, estimation performance, and general applicability (preamble or pilot-data-multiplexed setup, SISO or MIMO, with or without guardbands). Our MIMO pilot designs can also be extended to OFDMA downlink systems and OFDMbased cooperative communication systems. For OFDMA uplinks, the applicability of the proposed pilot designs depends on how resources are channelized for different users.

\section{REFERENCES}

[1] B. Razavi, RF Microelectronics. Upper Saddle River, NJ: Prentice-Hall, 1998.

[2] B. Razavi, "Design considerations for direct-conversion receivers," IEEE Trans. Circuits Syst. II, vol. 44, pp. 428-435, June 1997.

[3] C. L. Liu, "Impacts of I/Q imbalance on QPSK-OFDM-QAM detection," IEEE Trans. Consum. Electron., vol. 44, no. 3, pp. 984-989, Aug. 1998.

[4] M. Valkama, M. Renfors, and V. Koivunen, "Compensation of frequency-selective I/Q imbalances in wideband receivers: models and algorithms," in Proc. IEEE SPAWC, Mar. 2001, pp. 42-45.
[5] J. Tubbax, B. Come, L. Van der Perre, S. Donnay, M. Engels, M. Moonen, and H. De Man, "Joint compensation of IQ imbalance and frequency offset in OFDM systems," in Proc. Radio Wireless Conf. (RAWCON), 2003, pp. 39-42.

[6] A. Tarighat and A. H. Sayed, "Joint compensation of transmitter and receiver impairments in OFDM systems," IEEE Trans. Wireless Commun., vol. 6, no. 1, pp. 240-247, Jan. 2007.

[7] D. Tandur and M. Moonen, "Joint adaptive compensation of transmitter and receiver IQ imbalance under carrier frequency offset in OFDMbased systems," IEEE Trans. Signal Process., vol. 5, pp. 5246-5252, 2007.

[8] D. Tandur, C. Lee, and M. Moonen, "Efficient compensation of RF impairments for OFDM systems," in Proc. IEEE WCNC, Apr. 2009.

[9] F. Horlin, A. Bourdoux, and L. Van der Perre, "Low-complexity EMbased joint acquisition of the carrier frequency offset and IQ imbalance," IEEE Trans. Wireless Commun., vol. 7, pp. 2212-2220, 2008.

[10] I. Barhumi, G. Leus, and M. Moonen, "Optimal training design for MIMO OFDM systems in mobile wireless channels," IEEE Trans. Signal Process., vol. 51, no. 6, pp. 1615-1624, June 2003.

[11] H. Minn and N. Al-Dhahir, "Optimal training signals for MIMO OFDM channel estimation," IEEE Trans. Wireless Commun., vol. 5, no. 5, pp. 1158-1168, May 2006.

[12] H. Minn, N. Al-Dhahir, and Y. Li, "Optimal training signals for MIMO OFDM channel estimation in the presence of frequency offset and phase noise," IEEE Trans. Commun., vol. 54, no. 10, pp. 1754-1759, Oct. 2006.

[13] H. Minn, Y. Li, and N. Al-Dhahir, "PAR-constrained training signal designs for MIMO OFDM channel estimation in the presence of frequency offsets," IEEE Trans. Wireless Commun., vol. 7, no. 8, pp. 2884-2889, Aug. 2008

[14] X. Ma, L. Yang, and G. B. Giannakis, "Optimal training for MIMO frequency-selective fading channels," IEEE Trans. Wireless Commun., vol. 4, no. 2, pp. 453-466, Mar. 2005.

[15] W. Kirkland and K. Teo, "I/Q distortion correction for OFDM direct conversion receiver," Electron. Lett., vol. 39, pp. 131-133, 2003.

[16] L. Brotje, S. Vogeler, and K-D. Kammeyer, "Estimation and correction of transmitter-caused I/Q imbalance in OFDM systems," in Proc. 7th Intl. OFDM Workshop, Sep. 2002, pp. 178-182.

[17] Y. Egashira, Y. Tanabe, and K. Sato, "A novel IQ imbalance compensation method with pilot-signals for OFDM system," in Proc. IEEE VTC Fall, 2006, pp. 1-5.

[18] A. Tarighat, R. Bagheri, and A. Sayed, "Compensation schemes and performance analysis of IQ imbalances in OFDM receivers," IEEE Trans. Signal Process., vol. 53, pp. 3257-3268, 2005.

[19] R. Chrabieh and S. Soliman, "IQ Imbalance mitigation via unbiased training sequences," in Proc. IEEE GLOBECOM, 2007, pp. 4280-4285.

[20] E. Lopez-Estraviz, S. De Rore, F. Horlin, and L. Van der Perre, "Optimal training sequences for joint channel and frequency-dependent IQ imbalance estimation in OFDM-based receivers," in Proc. IEEE ICC, 2006, vol. 10, pp. 4595-4600.

[21] E. Lopez-Estraviz, S. De Rore, F. Horlin, and A. Bourdoux, "Pilot design for joint channel and frequency-dependent transmit/receive IQ imbalance estimation and compensation in OFDM-based transceivers," in Proc. IEEE ICC, 2007, pp. 4861-4866.

[22] Y. Chung and S. Phoong, "OFDM channel estimation in the presence of transmitter and receiver I/Q imbalance," in Proc. Euspico, 2008.

[23] T. Schenk, P. Smulders, and E. Fledderus, "Estimation and compensation of TX and RX IQ imbalance in OFDM-based MIMO systems," in Proc. IEEE Radio and Wireless Symposium, 2006, pp. 215-218.

[24] T. Schenk, P. Smulders, and E. Fledderus, "Estimation and compensation of frequency selective TX/RX IQ imbalance in MIMO OFDM systems," in Proc. IEEE ICC, 2006, vol. 1, pp. 251-256.

[25] Y. Zou, M. Valkama, and M. Renfors, "Digital compensation of I/Q imbalance effects in space-time coded transmit diversity systems," IEEE Trans. Signal Process., vol. 56, no. 6, pp. 2496-2508, June 2008.

[26] S. Kay, Fundamentals of Statistical Signal Processing. Prentice-Hall, 1993.

[27] Q. Gu, RF System Design of Transceivers for Wireless Communications. New York: Springer, 2005.

[28] A. Luzzatto and G. Shirazi, Wireless Transceiver Design. England: John Wiley \& Sons Ltd., 2007. 